\title{
FORMAS QUÍMICAS, DISPONIBILIDADE DE MANGANÊS E PRODUTIVIDADE DE SOJA EM SOLOS SOB SEMEADURA DIRETA ${ }^{(1)}$
}

\author{
Silvino Guimarães Moreira ${ }^{(2,3)}$, Luis Ignácio Prochnow ${ }^{(3)}$, Jorge de \\ Castro Kiehl $^{(3)}$, Ladislau Martin Neto ${ }^{(4)}$ \& Volnei Pauletti ${ }^{(5)}$
}

\begin{abstract}
RESUMO
O trabalho foi desenvolvido com os objetivos de testar, dentre vários extratores de micronutrientes do solo, qual é o mais adequado para avaliar a disponibilidade de Mn em solos sob sistema de semeadura direta (SSD); estudar, com auxílio da ressonância paramagnética eletrônica (RPE), a interação do Mn com os ácidos húmicos (AH) de solos de locais com e sem constatações de deficiência desse nutriente, e avaliar em quais das frações do solo (trocável, orgânica, óxido e residual) estava ligado o Mn, após sua aplicação no solo. Quatro locais foram selecionados para o estudo (Local I: Faz. Santa Rosa; II e III: Faz. Novo Horizonte e IV: Campo Experimental da Fundação ABC). Os Locais I, II e III situam-se em Tibagi, PR, e o Local IV, em Castro (PR). De modo geral, as doses de Mn aplicadas elevaram os teores de Mn nos solos, mas a concentração e a quantidade de Mn acumuladas pelas plantas de soja foram pouco influenciadas. Os teores de Mn nas frações trocável, orgânica e óxido das amostras do Local I aumentaram com as doses de Mn aplicadas no solo, tendo a maior elevação ocorrido na fração orgânica. Nessa fração, os teores aumentaram de $5,4 \mathrm{mg} \mathrm{kg}^{-1}$, na testemunha, para $35,1 \mathrm{mg} \mathrm{kg}^{-1}$, com a aplicação da maior dose. Os sinais de Mn não foram detectados por RPE nas amostras sólidas de AH (pH 3,0). A quantificação de Mn nessas amostras, após digestão nítrico-perclórica, juntamente com a observação de Mn por RPE, em amostras de AH em solução (pH 2,4), confirmou a hipótese de que o Mn presente nas amostras de AH estava na forma de ligações muito estáveis com os grupos funcionais (provavelmente carboxílicos) do AH. A ausência de efeito do Mn na produtividade da soja pode ser devida à complexação do nutriente pela MO. De forma geral, o DTPA-TEA foi o extrator mais adequado na avaliação da disponibilidade de Mn à soja.
\end{abstract}

Termos de indexação: plantio direto, extratores químicos, Mn.

\footnotetext{
(1) Parte da Tese de Doutorado do primeiro autor. Recebido para publicação em janeiro de 2003 e aprovado em fevereiro de 2005.

(2) Consultor Técnico do Recursos Humanos no Agronegócio - ReHAgro. Rua Mário Alves Teixeira 15, Centro, CEP 35710-000 Inhaúma (MG). E-mail: silvino@rehagro.com.br

(3) Professor do Departamento de Solos e Nutrição de Plantas, Escola Superior de Agricultura "Luiz de Queiroz" - ESALQ/USP. Caixa Postal 9, CEP 13418-900 Piracicaba (SP). E-mail: liprochnow@esalq.usp.br

(4) Pesquisador da Embrapa Instrumentação Agropecuária. Rua XV de Novembro 1452, CEP 13560-970 São Carlos (SP). E-mail: martin@cnpdia.embrapa.br

(5) Pesquisador de Fertilidade do Solo da Fundação ABC. BR 151, km 155,5, Caixa Postal 1003, CEP $84166-900$ Castro (PR). Email: vpauletti@fundaçãoabc.org.br
} 


\title{
SUMMARY: CHEMICAL FORMS IN SOILS AND BIOAVAILABILITY OF MANGANESE TO SOYBEAN UNDER NO-TILLAGE
}

\begin{abstract}
This field study had the following objectives: to determine the most appropriate micronutrient extractor to evaluate manganese bioavailability in soils under no-tillage; to evaluate the interaction of soil manganese with humic acids in soils under no-tillage with and without manganese deficiency through electronic paramagnetic resonance (EPR); and to evaluate manganese fractions (exchangeable, organic, oxides and residual) after manganese addition in soils under no-tillage with and without previous manganese deficiency for soybean. Four sites (I to IV) were selected to study the response curve to manganese ( 0 to $48 \mathrm{~kg} \mathrm{ha}^{-1}$ ) fertilization in soybean. Sites I to III were located in Tibagi, state of Paraná (PR), Brazil and site IV was in Castro (PR). Manganese rates affected the manganese concentrations in the soil, but not the manganese concentrations in leaves and the amount of manganese taken up by plants. Exchangeable, organic and oxide forms of manganese increased with the increasing rates applied at site I, with the largest increase being observed for the organic fraction (changed from $5.4 \mathrm{mg} \mathrm{kg}^{-1}$ manganese in the control to $35.1 \mathrm{mg} \mathrm{kg}^{-1}$ of manganese in the highest manganese dose). Manganese was not detected by EPR in the solid samples of humic acid-pH 3.0. Manganese quantification in the same samples after nitric-perchloric digestion, along clear

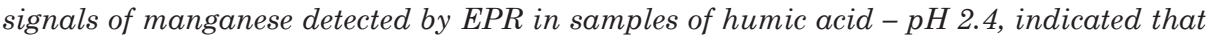
manganese in the samples was strongly linked to the functional (probably carboxylic) groups of humic acid. The fact that there was no effect of manganese on soybean yield might be due to the complexation of the nutrient by soil organic matter. In general, DTPA-TEA was the most reliable manganese extractor to evaluate manganese availability to soybean under no-tillage.
\end{abstract}

Index terms: No till, soil extractor, manganese, EPR.

\section{INTRODUÇÃO}

Em muitos casos, as deficiências de micronutrientes são atribuídas apenas ao aumento de $\mathrm{pH}$ do solo, causado pela calagem excessiva, especialmente em virtude da má incorporação e, ou, aplicação de doses de calcário acima da recomendada nos solos cultivados sob sistema de semeadura convencional (SSC) (Tanaka et al., 1993; Sanzonowicz, 1995). Em locais sob sistema de semeadura direta (SSD), existem alguns relatos de "manchas" de deficiências de Mn, causadas pela má distribuição do corretivo aplicado superficialmente (Motta \& Prevedello, 1995). Contudo, essas explicações nem sempre procedem para muitos solos sob semeadura direta, uma vez que, embora apresentem teores adequados ou elevados de Mn, de acordo com o método oficial, tais solos vêm revelando deficiências de Mn em soja (Pauletti, 1998).

A tendência de os teores de Mn "trocáveis" da camada superficial serem mais elevados em solos sob SSD, em relação ao SSC (Castro et al., 1992; Edwards et al., 1992; Motta \& Prevedello, 1995; Motta et al., 2002), não pode ser explicada apenas pelos valores de $\mathrm{pH}$ do solo. No SSD, a aplicação superficial de calcário resulta em valores de $\mathrm{pH}$ mais elevados em superfície (Caires et al., 1999; Caires et al., 2000; Moreira et al., 2001), o que, em conseqüência, deveria diminuir e não aumentar os teores desse nutriente. Vários autores têm atribuído as maiores quantidades de Mn no SSD aos teores mais elevados de MO existentes nos solos submetidos a esse sistema de cultivo (Castro et al., 1992; Edwards et al., 1992; Motta et al., 2002).

Em solos norte-americanos e africanos, a aplicação de elevadas doses de fertilizantes nitrogenados em locais com predomínio de gramíneas nas rotações de culturas e, ou, monocultivos de milho tem causado diminuição dos valores de $\mathrm{pH}$ e aumentos dos teores de Mn da camada superficial (Follett \& Peterson, 1988; Grove \& Blevins, 1988; Juo et al., 1995; Bowman \& Halvorson, 1998) e, conseqüentemente, elevação da concentração foliar. No entanto, em condições brasileiras, a acidez da camada superficial dos solos sob SSD geralmente não é elevada, principalmente por causa da calagem superficial e do emprego de rotações e, ou, sucessões que utilizam leguminosas e evitam a monocultura de milho. Os valores de $\mathrm{pH}_{\mathrm{CaCl} 2}$ encontrados na camada de 0-5 cm de três solos estudados por Moreira et al. (2001) estavam todos acima de 5,0, mesmo antes da calagem.

Essas constatações evidenciam que os teores de Mn extraídos do solo não estão disponíveis às plantas, mas ligados à MO. Desse modo, os extratores desenvolvidos para solos sob SSC não demonstram a capacidade de discriminar a quantidade disponível em solos sob SSD. 
O fracionamento químico ou a extração seqüencial de micronutrientes (formas solúvel + trocável, orgânica, óxido e residual) e o posterior estudo de correlação entre os teores de micronutrientes distribuídos nessas formas com a quantidade absorvida pela planta podem auxiliar os estudos de seleção de extratores químicos mais adequados na avaliação da disponibilidade de micronutrientes. Utilizando a ressonância paramagnética eletrônica (RPE), é possível, em alguns casos, estudar a forma pela qual os metais estão complexados organicamente.

Diante do exposto, desenvolveu-se o estudo com os seguintes objetivos: 1- avaliar diferentes extratores de micronutrientes do solo para: (a) medir a disponibilidade de Mn em solos sob sistema de semeadura direta (b) identificar fração do solo (trocável, orgânica, óxido e residual) com a que o Mn predominantemente se associa depois de sua aplicação; 2- estudar, com o auxílio da técnica da ressonância paramagnética eletrônica, a interação do Mn com os ácidos húmicos dos solos de locais com e sem constatações de deficiências desse nutriente.

\section{MATERIAL E MÉTODOS}

O estudo foi desenvolvido na Região Centro Sul do Paraná, utilizando-se quatro experimentos em solos sob SSD com ampla variação nos teores de Mn, uma vez que os resultados preliminares obtidos por Moreira (2003) indicaram que uma das causas da ausência de correlação entre os teores de Mn no solo (DTPA-TEA, Mehlich-1 e 3, HCl 0,1 mol L-1) e a concentração na planta foi a pequena amplitude dos dados. Os atributos dos solos utilizados encontramse nos quadros 1 e 2 , respectivamente. Os atributos do solo foram avaliados de acordo com o método descrito por Raij et al. (1987). Detalhes sobre o histórico de cultivo dos quatro locais são apresentados no quadro 3. Em dois dos locais (I e II), têm sido constatadas deficiências de Mn em soja, enquanto os outros dois não apresentam histórico de deficiência.

As amostras de terra de todos os locais, antes da aplicação das doses de Mn, foram submetidas à extração seqüencial de Mn. O método empregado foi adaptado de Chang et al. (1984), Shuman (1985) e Ahnstrom \& Parker (1999), conforme descrito a seguir: As formas (trocável + solúvel) foram extraídas com solução de nitrato de estrôncio $0,1 \mathrm{~mol} \mathrm{~L}^{-1}$; micronutrientes ligados à fração orgânica com solução de hipoclorito de sódio a 5 dag $\mathrm{L}^{-1}$ ou cL L L $\mathrm{L}^{-1}$, com $\mathrm{pH}$ ajustado a 8,5; fração ligada aos óxidos de ferro, alumínio e Mn pela mistura das soluções de ácido oxálico 0,2 mol $\mathrm{L}^{-1}+$ oxalato de amônio $0,2 \mathrm{~mol} \mathrm{~L}^{-1}+$ ácido ascórbico $0,1 \mathrm{~mol} \mathrm{~L}^{-1}$, ajustada a $\mathrm{pH} 3,0$; e fração residual e teores totais por digestão ácida em microondas, utilizando os ácidos clorídrico, nítrico e fluorídrico concentrados.

Foi feita a extração das substâncias húmicas, bem como a separação dos ácidos húmicos $(\mathrm{AH})$ de acordo com a técnica de extração, fracionamento e purificação recomendada por Schnitzer (1982), sem utilização do $\mathrm{Na}_{4} \mathrm{P}_{2} \mathrm{O}_{7} 0,1 \mathrm{~mol} \mathrm{~L}^{-1}$, uma vez que sua recomendação é restrita aos solos com elevados teores de Ca (Alexandrova, 1960). As análises dos $\mathrm{AH}$ sólidos e em solução ( $\mathrm{pH} 3,0$ e 2,5), por RPE, foram realizadas na Embrapa/CNPDIA, conforme descrito por Moreira (2003). Os AH foram digeridos (digestão nítrico-perclórica) para obtenção dos teores de Mn.

Para obter a amplitude desejada dos teores de Mn no solo e na planta, utilizaram-se sete doses de Mn $\left(0 ; 1,5 ; 3 ; 6 ; 12 ; 24\right.$ e $\left.48 \mathrm{~kg} \mathrm{ha}^{-1}\right)$, na forma de $\mathrm{MnSO}_{4} \cdot \mathrm{H}_{2} \mathrm{O}(31 \%$ de $\mathrm{Mn}$ ), aplicadas manualmente, a lanço, no dia 23/10/2001, antes da semeadura da soja. Para equalizar o S aplicado na forma de $\mathrm{MnSO}_{4} \cdot \mathrm{H}_{2} \mathrm{O}$, utilizou-se $\mathrm{S}$ na forma elementar. Considerando os baixos teores de $\mathrm{B}$, foram aplicados em todos os locais $2 \mathrm{~kg} \mathrm{ha}^{-1}$ do nutriente a lanço, na

Quadro 1. Atributos dos solos na camada de 0,00-0,10 m

\begin{tabular}{|c|c|c|c|c|c|c|c|c|c|c|c|c|c|}
\hline $\begin{array}{l}\text { Local/Def. de } \\
\text { manganês( }\end{array}$ & $\mathrm{pH} \mathrm{CaCl}{ }_{2}$ & $\mathbf{P}$ & $\mathrm{S}_{-\mathrm{SO}_{4}-2}$ & MO & $\mathbf{K}$ & $\mathbf{C a}$ & Mg & $\mathrm{V}$ & B & $\mathrm{Cu}$ & $\mathbf{F e}$ & Mn & $\mathrm{Zn}$ \\
\hline & & \multicolumn{2}{|c|}{$-\mathrm{mg} \mathrm{dm}^{-3}{ }_{-}$} & $\mathrm{g} \mathrm{dm}^{-3}$ & \multicolumn{3}{|c|}{$-\mathrm{mmol}_{\mathrm{c}} \mathrm{dm}^{-3}-$} & $\%$ & 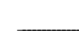 & 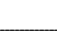 & $\mathrm{g} \mathrm{dm}$ & 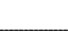 & - \\
\hline I- Com Def. & 5,2 & 38 & 11 & 47 & 3,8 & 41 & 23 & 62 & 0,19 & 1,0 & 98 & 1,2 & 1,5 \\
\hline II- Com Def. & 5,5 & 24 & 14 & 41 & 3,4 & 56 & 24 & 75 & 0,14 & 2,0 & 103 & 1,4 & 0,5 \\
\hline III- Sem Def. & 5,4 & 34 & 11 & 29 & 3,6 & 66 & 12 & 74 & 0,12 & 1,0 & 47 & 3,5 & 1,7 \\
\hline IV-Sem Def. & 5,8 & 133 & 9 & 53 & 4,2 & 77 & 30 & 82 & nd & 2,2 & 42 & 3,9 & 8,2 \\
\hline
\end{tabular}

(1) Local I: Faz. Santa Rosa; Locais II e III: Faz. Novo Horizonte; Local IV: Campo Experimental da Fundação ABC. Com Def.: local com constatação de deficiência de manganês em soja; Sem Def.: local onde não é constatada deficiência. Os locais I, II e III estão localizados no município de Tibagi, e o Local IV, em Castro (PR). nd: teor não detectado. Os atributos do solo foram avaliados de acordo com o método descrito por Raij et al. (1987). 
Quadro 2. Conteúdos de areia, silte e argila totais, na camada de 0-0,10 m

\begin{tabular}{ccccc}
\hline Local/Def. de manganês(1) & Areia & Silte & Argila & Classificação textural \\
\cline { 3 - 4 } & & g kg-1 $^{(1)}$ & & \\
I- Com Def. & 770 & 20 & 210 & média arenosa \\
II- Com Def. & 660 & 40 & 300 & média argilosa \\
III- Sem Def. & 710 & 20 & 270 & média argilosa \\
IV- Sem Def. & 210 & 90 & 700 & muito argilosa
\end{tabular}

(1) Local I: Faz. Santa Rosa; Locais II e III: Faz. Novo Horizonte; Local IV: Campo Experimental da Fundação ABC. Com Def.: local com constatação de deficiência de manganês em soja; Sem Def.: local onde não é constatada deficiência. Os locais I, II e III estão localizados no município de Tibagi, e o Local IV, em Castro (PR).

Quadro 3. Histórico de cultivo dos locais I, II, III e IV

\begin{tabular}{|c|c|}
\hline Local & Característica \\
\hline $\begin{array}{c}\text { Faz. Santa Rosa } \\
\text { (Local I) }\end{array}$ & $\begin{array}{l}\text { Área de integração agricultura/pecuária, sendo dois anos consecutivos de soja, seguido de um } \\
\text { ano de milho no verão. No inverno, foram semeadas a lanço sementes de azevém }\left(30 \mathrm{~kg} \mathrm{ha}^{-1}\right) \text {, } \\
\text { misturadas com aveia preta }\left(50 \mathrm{~kg}^{-1} \text { ) para pastejo de gado. O solo (Cambissolo Húmico }\right. \\
\text { distrófico típico textura média) foi cultivado em SSD por mais de oito anos tendo sido } \\
\text { constatadas constantes deficiências de manganês em soja. Realizou-se a última calagem no } \\
\text { inverno de } 1999 \text {, empregando-se } 2.000 \mathrm{~kg} \mathrm{ha}^{-1} \text { superficialmente. }\end{array}$ \\
\hline $\begin{array}{c}\text { Faz. Novo } \\
\text { Horizonte (Locais } \\
\text { II e III) }\end{array}$ & $\begin{array}{l}\text { Área com rotação soja e milho, sendo dois anos consecutivos de soja e um ano de milho. No } \\
\text { inverno, foi utilizada aveia preta para produção de massa, com exceção do ano de } 2001 \text {, em que } \\
\text { foi utilizado trigo. Os dois locais (II e III) foram cultivados em SSD por cinco anos. O solo do } \\
\text { local II foi classificado como Cambissolo Háplico Tb distrófico típico A proeminente textura } \\
\text { média tendo sido observadas constantes deficiências de manganês em soja nos últimos anos. O } \\
\text { solo do local III é um Cambissolo Húmico distrófico típico textura média e no local não foram } \\
\text { observadas deficiências de manganês. No inverno de } 2000 \text {, foi realizada a última calagem, } \\
\text { empregando-se } 2000 \mathrm{~kg} \mathrm{ha}^{-1} \text { superficialmente. }\end{array}$ \\
\hline $\begin{array}{c}\text { Campo } \\
\text { Experimental } \\
(\text { Local IV) }\end{array}$ & $\begin{array}{l}\text { Área com rotação milho e soja, no verão, e aveia preta, no inverno. Local cultivado sob SSD } \\
\text { desde de } 1998 \text { e sem constatação de deficiência de manganês em soja. O solo foi um Latossolo } \\
\text { Bruno distrófico típico A proeminente textura muito argilosa. A última calagem foi realizada } \\
\text { no inverno de } 1997 \text {, empregando-se } 2.000 \mathrm{~kg} \mathrm{ha}^{-1} \text { superficialmente. }\end{array}$ \\
\hline
\end{tabular}

forma de ácido bórico (17 \% de B). Pela mesma razão, foram aplicados no Local II $5 \mathrm{~kg} \mathrm{ha}^{-1}$ de Zn, a lanço, na forma de sulfato de zinco heptaidratado $(20 \%$ de $\mathrm{Zn})$. Cada experimento foi conduzido em delineamento experimental de blocos ao acaso (quatro blocos). O tamanho das parcelas correspondeu a $2,8 \times 8 \mathrm{~m}$ de comprimento $\left(22,4 \mathrm{~m}^{2}\right)$.

A semeadura da soja (cv. BRS 133) foi efetuada no dia 23/10/2001 nos locais II e III; no dia 31/10/ 2001, no Local I; e em 01/11/2001, no Local IV. Nos locais I e IV, utilizou-se o espaçamento de 0,40 m entre linhas e, nos demais, 0,45 m. Na adubação de plantio dos locais I, II e III, aplicaram-se $250 \mathrm{~kg} \mathrm{ha}^{-1}$ da fórmula 0-25-25 em razão da menor fertilidade dos solos nesses locais; e no Local IV, empregaramse $100 \mathrm{~kg} \mathrm{ha}^{-1} \mathrm{da}$ fórmula 0-26-26. Detalhes sobre adubação de plantio, tratamento de sementes, controle de plantas invasoras, pragas e doenças podem ser vistos em Moreira (2003).

As amostras de terra e de plantas foram coletadas no dia 23/01/2002, quando a soja dos locais II, III e IV encontrava-se no início do florescimento (estádio - R2), e a soja do Local I, no estádio imediatamente anterior ao estádio reprodutivo, $\mathrm{R} 1$. As amostras de terra foram retiradas com trado calador na profundidade de 0,00-0,10 m, em número de 20 amostras simples por parcela. Estas amostras foram secas em estufa a $40^{\circ} \mathrm{C}$, destorroadas, moídas e passadas em peneira de $2 \mathrm{~mm}$. Nessas amostras, foram determinados os teores de $\mathrm{Mn}$ com quatro extratores: 1- Solução de ácido dietileno-triaminopentacético (DTPA) 0,005 $\mathrm{mol} \mathrm{L}^{-1}+$ trietanolamina (TEA) $0,1 \mathrm{~mol} \mathrm{~L}^{-1}+\mathrm{CaCl}_{2}$ 0,01 $\mathrm{mol} \mathrm{L}^{-1}$ a pH 7,3 (Lindsay \& Norvell, 1978); 2- Mehlich-1 (Solução de 
$\mathrm{HCl}$ 0,05 mol L-1 $+\mathrm{H}_{2} \mathrm{SO}_{4}$ 0,0125 $\mathrm{mol} \mathrm{L}^{-1}$ ) (Embrapa, 1979); 3- Mehlich-3 (Solução de $\mathrm{CH}_{3} \mathrm{COOH} \mathrm{0,2} \mathrm{\textrm {mol } \mathrm { L } ^ { - 1 } +}$ $\mathrm{NH}_{4} \mathrm{NO}_{3} 0,25 \mathrm{~mol} \mathrm{~L}^{-1}+\mathrm{NH}_{4} \mathrm{~F} 0,015 \mathrm{~mol} \mathrm{~L}^{-1}+\mathrm{HNO}_{3}$ 0,013 $\mathrm{mol} \mathrm{L}^{-1}+$ EDTA 0,001 $\mathrm{mol} \mathrm{L}^{-1}$ ) (Mehlich, 1984) e solução de $\mathrm{HCl}$ 0,1 mol L-1 (Wear \& Sommer, 1948).

Nas amostras dos tratamentos 0,12 e $48 \mathrm{~kg} \mathrm{ha}^{-1}$ de Mn do Local I, foi feita a extração seqüencial de Mn. Também foram extraídas amostras de AH dos locais I e II, das parcelas que receberam $48 \mathrm{~kg} \mathrm{ha}^{-1}$ de $\mathrm{Mn}$, conforme método descrito anteriormente. Em amostras sólidas de AH e em solução ( $\mathrm{pH}$ 3,0 e $2,5)$, foram avaliadas por RPE as interações dos metais com os grupos funcionais dos $\mathrm{AH}$, conforme método descrito por Moreira (2003).

No caso das amostras de plantas, coletaram-se duas amostras por parcela (parte aérea) de plantas de $0,5 \mathrm{~m}$ de sulco cada. Após a coleta, as amostras foram lavadas em água deionizada, colocadas para secar em estufa a $65^{\circ} \mathrm{C}$ até atingir massa constante, pesadas e moídas. Nessas amostras, foram analisadas as concentrações de Mn, conforme Malavolta et al. (1997).

\section{RESULTADOS E DISCUSSÃO}

A partir dos resultados da extração seqüencial do Mn, observou-se que os solos mais argilosos (locais II e IV) apresentaram maiores teores totais de $\mathrm{Mn}$, em relação aos mais arenosos (Quadro 4). Resultados similares foram observados por Valadares \& Camargo (1983) e Nascimento et al. (2002), em solos dos estados de São Paulo e Minas Gerais, respectivamente. A faixa de teores encontrada por Valadares \& Camargo (1983) foi de 14 a $2.395 \mathrm{mg} \mathrm{kg}^{-1}$ em solos arenosos e latossolos, respectivamente. Por sua vez, os teores naturais totais obtidos por Nascimento et al. (2002) variaram de 49 a $1.160 \mathrm{mg} \mathrm{kg}^{-1}$, sendo os maiores teores encontrados nos Latossolos originados de rochas máficas, e os menores, nos derivados de granitos e gnaisses.

Os teores totais de Mn, extraídos das amostras de terra dos locais I (42,4 $\left.\mathrm{mg} \mathrm{kg}^{-1}\right)$ e III (44,8 $\left.\mathrm{mg} \mathrm{kg}^{-1}\right)$ estão próximos dos menores valores encontrados por Nascimento et al. (2002). Acredita-se que, após inúmeros cultivos em solos com baixos teores totais de Mn, as plantas têm maior probabilidade de apresentarem deficiências do que aquelas cultivadas em solos com altos teores iniciais, em razão do esgotamento natural dos solos. Deste modo, outro problema de alguns solos sob SSD da Região CentroSul do Paraná, principalmente os arenosos, é a baixa fertilidade natural em Mn, que, associada à calagem superficial e ao aumento do teor de MO causado pelo SSD, potencializa a possibilidade de ocorrência de deficiências.

A partir da extração seqüencial do $\mathrm{Mn}$ das amostras de terra do Local I, realizada após três meses da aplicação do nutriente no solo (doses 0, 12 e $48 \mathrm{~kg} \mathrm{ha}^{-1}$ ), verificou-se que os teores de $\mathrm{Mn}$ trocáveis, extraídos com $\mathrm{Sr}\left(\mathrm{NO}_{3}\right)_{2}$, aumentaram com as doses aplicadas (Figura 1). Os teores elevaramse de 0,6 mg kg-1, na testemunha, para $2,5 \mathrm{mg} \mathrm{kg}^{-1}$, com a aplicação da maior dose (Quadro 5). Esse aumento, apesar de ter sido mais de quatro vezes superior ao teor original, foi pequeno, quando comparado com o aumento ocorrido na fração orgânica.

$\mathrm{Na}$ fração orgânica, os teores de Mn variaram de $5,4 \mathrm{mg} \mathrm{kg}^{-1}$, na testemunha, para $35,1 \mathrm{mg} \mathrm{kg}^{-1}$, com a maior dose. Nas parcelas sem aplicação de Mn, $44,2 \%$ do Mn (soma das frações) encontrava-se na forma residual e apenas 15,5 \% na forma orgânica. Com a aplicação de $48 \mathrm{~kg} \mathrm{ha}^{-1}$ de $\mathrm{Mn}$, a quantidade de Mn na forma orgânica passou para 51,9 \% do Mn total, e apenas $20 \%$ ficou na forma residual, demonstrando que a maior parte do Mn ficou retido na MO do solo.

Quadro 4. Teores de manganês na camada de 0,00-0,10 m de profundidade, em diferentes frações do solo, nos locais com e sem deficiência de manganês

\begin{tabular}{|c|c|c|c|c|c|c|}
\hline \multirow{2}{*}{$\begin{array}{l}\text { Local/Def. de } \\
\text { manganês(1) }\end{array}$} & \multicolumn{4}{|c|}{ Frações } & \multirow{2}{*}{ Soma } & \multirow{2}{*}{ Total } \\
\hline & Trocável & Orgânica & Óxidos & Residual & & \\
\hline I- Com Def. & $0,9(2,6)^{2}$ & $8,4(23,1)$ & $13,4(36,8)$ & $13,6(37,5)$ & 36,3 & 42,4 \\
\hline II- Com Def. & $0,9(0,4)$ & $12,7 \quad(5,3)$ & $69,9(29,1)$ & $156,4(65,2)$ & 239,8 & 228,3 \\
\hline III- Sem Def. & $2,2(3,5)$ & $10,1(15,9)$ & $43,5(68,9)$ & $7,4(11,6)$ & 63,2 & 44,8 \\
\hline IV-Sem Def. & $0,9(0,9)$ & $28,9(29,3)$ & $38,4(39,0)$ & $30,4(30,8)$ & 98,5 & 85,3 \\
\hline
\end{tabular}

\footnotetext{
(1) Local I: Faz. Santa Rosa; Locais II e III: Faz. Novo Horizonte; Local IV: Campo Experimental da Fundação ABC. Com Def.: local com constatação de deficiência de manganês em soja; Sem Def.: local onde não é constatada deficiência. Os locais I, II e III estão localizados no município de Tibagi, e o Local IV, em Castro (PR). ${ }^{(2)}$ Valores entre parênteses referem-se à percentagem do teor em relação à soma das frações.
} 


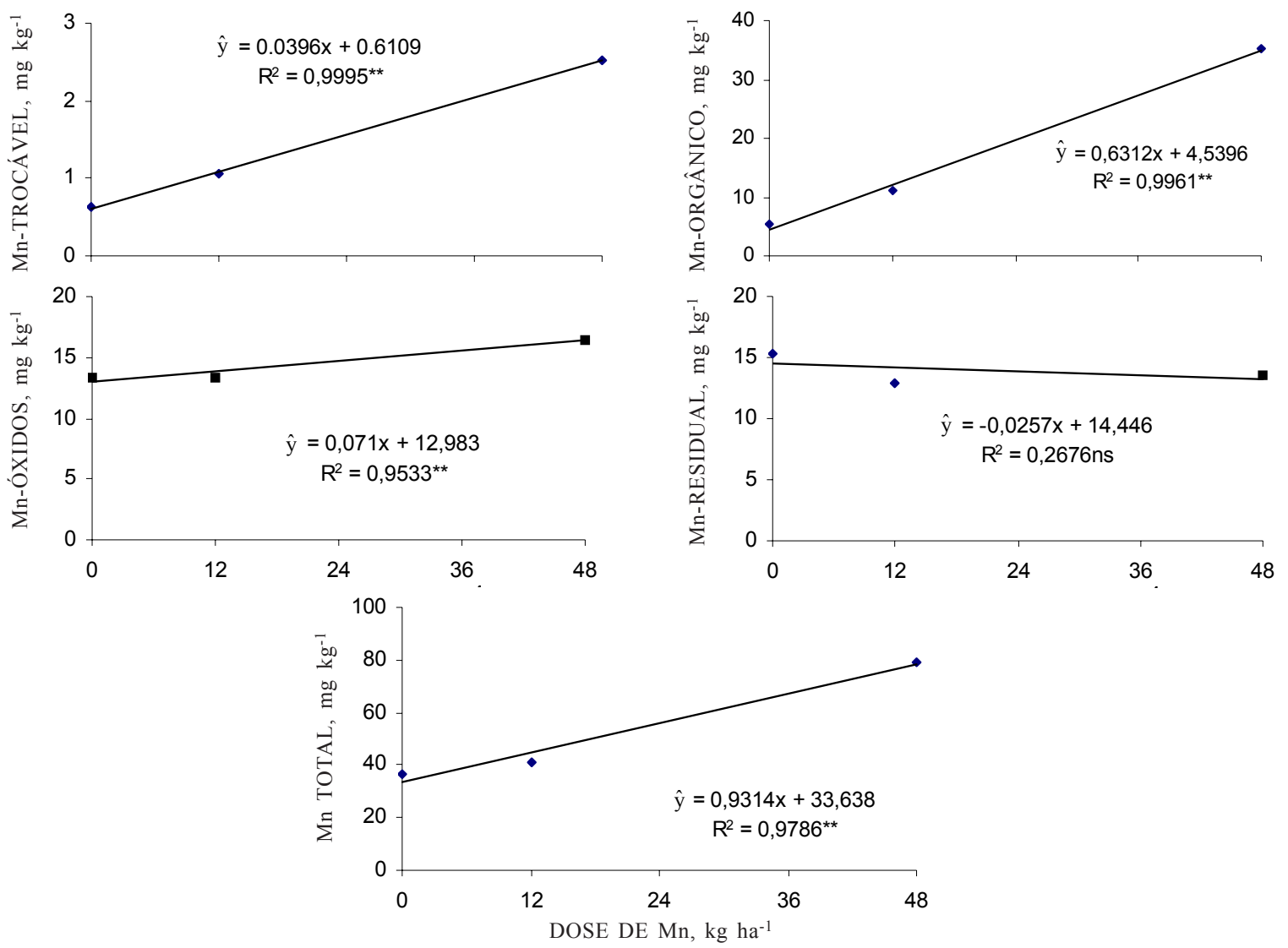

Figura 1. Formas de manganês extraídas do Cambissolo Húmico do Local I (Faz. Santa Rosa), considerando as doses de manganês aplicadas. Os dados originais apresentados foram transformados em $\mathrm{x}^{1 / 2} \mathrm{para} \mathrm{as}$ análises estatísticas. ** significativo a $1 \%$.

Quadro 5. Teores de manganês em diferentes formas, na camada de 0,00-0,10 m de profundidade, considerando as doses de manganês aplicadas no Local I (Faz. Santa Rosa)

\begin{tabular}{|c|c|c|c|c|c|c|}
\hline \multirow{2}{*}{ Dose } & \multicolumn{4}{|c|}{ Frações } & \multirow{2}{*}{$\begin{array}{c}\text { Soma das } \\
\text { frações }\end{array}$} & \multirow{2}{*}{ Total } \\
\hline & Trocável & Orgânica & Óxidos & Residual & & \\
\hline \multicolumn{7}{|l|}{$\mathrm{kg} \mathrm{ha-1}$} \\
\hline 0 & $0,6\left(1,8^{*}\right) \mathrm{C}^{1}$ & $5,4(15,5) \mathrm{B}$ & $13,3(38,4) \mathrm{A}$ & $15,3(44,2) \mathrm{A}$ & 34,6 & 36,5 \\
\hline 12 & $1,1(2,6) \mathrm{B}$ & $11,0(28,7) \mathrm{A}$ & $13,4(34,9) \mathrm{A}$ & $13,0(33,9) \mathrm{A}$ & 38,4 & 41,0 \\
\hline 48 & $2,5(3,7) \mathrm{C}$ & $35,1(51,9) \mathrm{A}$ & $16,5(24,4) \mathrm{B}$ & $13,5(20,0) \mathrm{B}$ & 67,6 & 79,3 \\
\hline C.V. (\%) & 6,9 & & & & & \\
\hline
\end{tabular}

\footnotetext{
* Valores dentro dos parênteses referem-se às percentagens dos teores em relação à soma das frações. Os dados originais apresentados foram transformados $\mathrm{em}^{1 / 2}$ para as análises estatísticas.

(1) Letras maiúsculas comparam, na linha, teores de manganês nas diferentes frações, dentro de cada dose, pelo teste de Tukey a $5 \%$.
}

O Mn retido pela $\mathrm{MO}$ pode estar associado aos grupos funcionais da mesma, na forma de complexos de esfera externa e interna. No caso dos complexos de esfera externa, o Mn é adsorvido apenas por atração eletrostática (Lakatos et al., 1977), permanecendo solvatado às moléculas de água, $\left[\mathrm{Mn}\left(\mathrm{H}_{2} \mathrm{O}\right)_{6}\right]^{2+}$ (Novotny, 2002). Esse tipo de interação é relativamente fraca e o metal complexado pode ser facilmente trocado por outros cátions não-específicos (Evangelou \& Marsi, 2001) e encontra-se disponível às plantas (Canellas et al., 1999). 
Quando as moléculas de água, que solvatam o cátion são trocadas pelos grupamentos funcionais da MO, e o cátion passa a se coordenar diretamente aos grupos funcionais, por meio de ligações covalentes mais fortes que aquelas existentes entre o Mn e as moléculas de $\mathrm{H}_{2} \mathrm{O}$ no íon $\left[\mathrm{Mn}\left(\mathrm{H}_{2} \mathrm{O}\right)_{6}\right]^{2+}$, é formado um complexo chamado esfera interna (Canellas et al., 1999). Nesses complexos, forma-se um quelato em que o ligante precisa ter, no mínimo, dois centros doadores de elétrons, capazes de se ligar ao mesmo íon metálico, formando-se um anel ou pinça denominado quelato (Evangelou \& Marsi, 2001). Os principais grupos funcionais envolvidos na quelatilização seriam o carboxílico, as hidroxilas fenólicas e alcoólica, a carbonila e a metoxila (Schnitzer \& Khan, 1978; Novotny, 2002). Desses grupos, o carboxílico é o mais importante (Evangelou \& Marsi, 2001).

$\mathrm{O} \mathrm{Mn}^{2+}$ adsorvido pela MO em baixos valores de $\mathrm{pH}$, provavelmente está presente nos solos na forma de complexos de esfera externa $\left(\left[\mathrm{Mn}\left(\mathrm{H}_{2} \mathrm{O}\right)_{6}\right]^{2+}\right)$, enquanto grande parte pode estar como complexo de esfera interna (quelatos), com o aumento do $\mathrm{pH}$ e temperatura (McBride, 1982; Senesi, 1990). Com a elevação do pH, aumenta-se a ionização (desprotonação) dos grupos carboxílicos presentes nos AH e ácido fúlvico (AF) (Evangelou \& Marsi, 2001) e, conseqüentemente, sua tendência em se ligar ao Mn (Bibak, 1994; Stevenson, 1994). Nos solos com altos valores de $\mathrm{pH}$, a atividade dos microrganismos que oxidam o $\mathrm{Mn}^{2+}$ em complexos estáveis com a MO também é mais elevada (Tisdale et al., 1985).

Em virtude dos altos teores de MO dos solos sob SSD (Bayer et al., 2002a,b; Motta et al., 2002), das fortes correlações entre os teores de Mn e a MO
(Castro et al., 1992; Motta et al., 2002) e das deficiências de Mn em soja nos solos sob SSD, com teores considerados adequados para o SSC, provavelmente parte do Mn extraído desses solos pode estar associada aos grupos funcionais da MO em formas muito estáveis e indisponíveis às plantas. Apesar de as evidências apontarem para esse fato, somente a partir dos dados obtidos pela extração seqüencial, não é possível inferir sobre o tipo de complexo MO-metal. O extrator utilizado para extração do $\mathrm{Mn}$ da forma orgânica $(\mathrm{NaClO})$ oxida praticamente $100 \%$ da MO do solo (Chao, 1984), liberando para a solução todo o Mn associado à MO, em diferentes formas (atração eletrostática, ligação covalente). Para determinar as formas de ligações do Mn com a MO, outras técnicas devem ser utilizadas, como, por exemplo, a técnica da ressonância paramagnética eletrônica (RPE).

Utilizando a RPE, inicialmente, não foram obtidos sinais de $\mathrm{Mn}^{2+}$ nas amostras de AH (Figuras 2 e 3), mediante o alargamento das linhas provocado pelo $\mathrm{Fe}^{3+}$ (Ceretta et al., 1999), o qual se encontra nas formas de óxido e hidróxido (Novotny, 2002). A centrifugação das amostras de $\mathrm{AH}$, em alta rotação, foi altamente eficiente na eliminação do ferro (Figuras 2 e 3), respectivamente, para as amostras de AH, extraídas dos solos dos locais I e III. O alargamento da linha acontece porque o íon $\mathrm{Fe}^{3+}$ diminui os tempos de relaxação dos spins dos elétrons, causando a saturação do sinal (Saab, 1999) e, conseqüentemente, perda de informações.

Nos espectros de RPE, apareceu outro sinal de $\mathrm{Fe}^{3+}$, que, geralmente, apresenta fator g de $4,3 \mathrm{e}$ simetria rômbica (Novotny, 2002). Esse ferro, ligado aos radicais orgânicos, mostra considerável

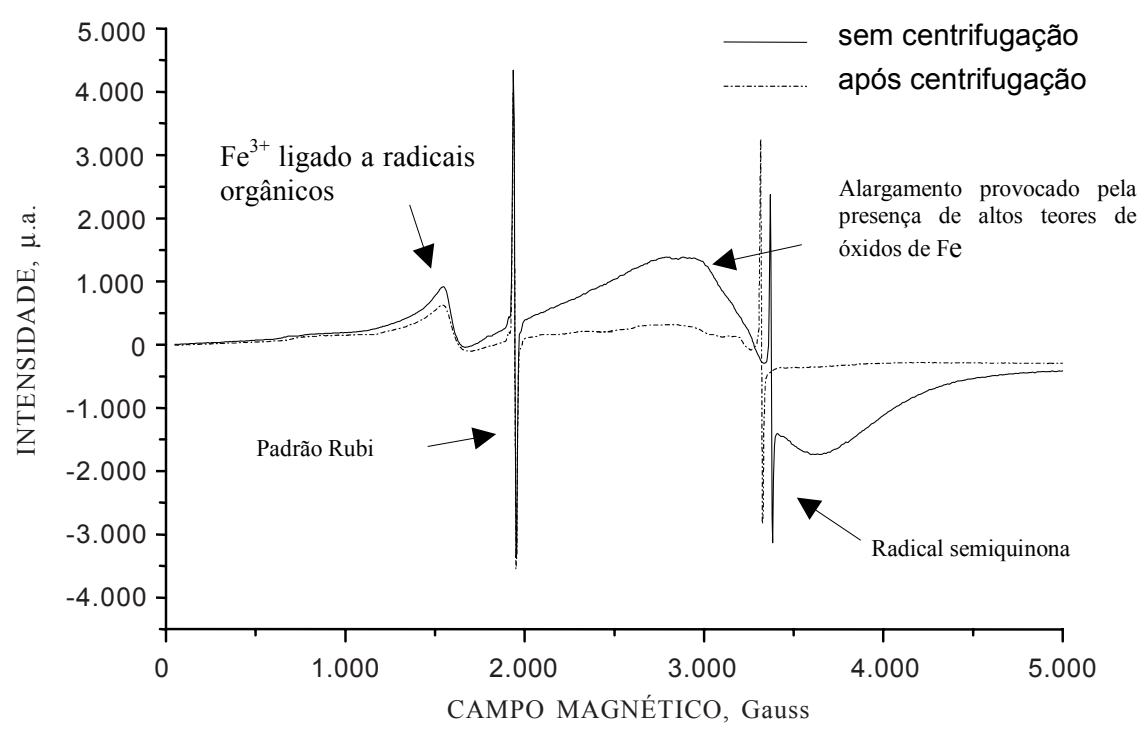

Figura 2. Espectro de RPE de amostras sólidas de AH, extraído da camada de 0,00-0,10 m, do Local I, antes e depois da centrifugação de $12.500 \mathrm{rpm}$ por $3 \mathrm{~h}$. $\mathrm{I}=$ intensidade. 


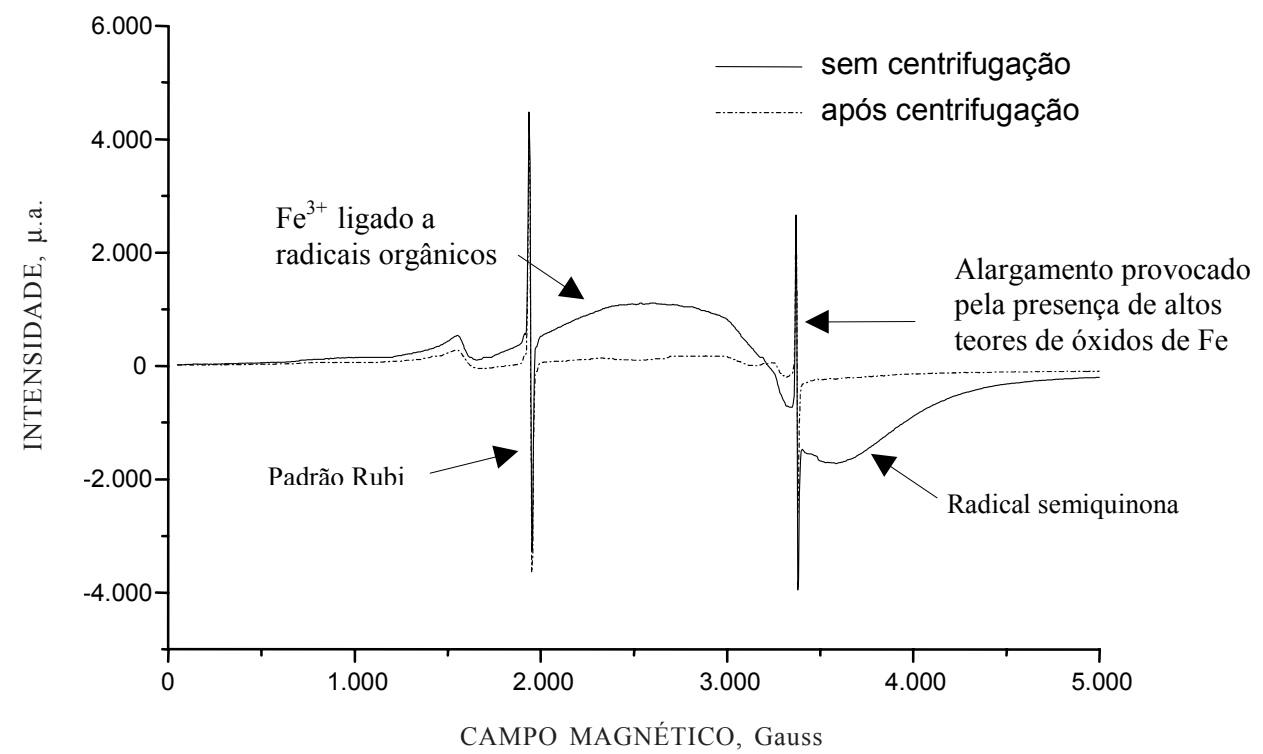

Figura 3. Espectro de RPE de amostras sólidas de AH, extraído da camada de 0,00-0,10 m, do Local III, antes e depois da centrifugação de $12.500 \mathrm{rpm}$ por $3 \mathrm{~h}$. I = intensidade.

resistência em reduzir ou realizar reações de troca, por ser fortemente ligado e protegido, na forma de complexos de esfera interna com os AH (Saab, 1999). Prova disso é que os sinais do $\mathrm{Fe}^{3+}$, associado aos radicais orgânicos, persistiram após o tratamento por centrifugação.

Mesmo após a eliminação do ferro das amostras de $\mathrm{AH}$, os sinais do $\mathrm{Mn}$ ainda não puderam ser obtidos (Figuras 2 e 3), provavelmente porque a quantidade remanescente de ferro deve ter sido alta o suficiente para interferir nos sinais de Mn. Além disso, é possível que os processos de extração e purificação dos AH, juntamente com a centrifugação para eliminação dos óxidos de ferro, tenham eliminado grande parte do Mn e diminuído sua quantidade adsorvida à MO. Senesi \& Sposito (1989) observaram que os sinais de RPE do $\mathrm{Mn}^{2+}$, em amostras de liteira, desapareceram após sucessivos tratamentos ácidos das amostras.

Durante o processo de extração e fracionamento das substâncias húmicas do solo, a separação dos $\mathrm{AH}$ e AF é feita por meio da precipitação dos primeiros em condições ácidas (pH 1,0 a 1,5). Esse abaixamento do $\mathrm{pH}$ provoca a liberação dos metais associados aos AH para os AF em solução. Desse modo, em cada etapa de abaixamento do $\mathrm{pH}$, para precipitação dos $\mathrm{AH}$, a quantidade de metais adsorvida aos $\mathrm{AH}$ é diminuída, e a dos $\mathrm{AF}$, aumentada. Acredita-se que tanto os metais associados fracamente aos $\mathrm{AH}$, como parte daqueles ligados de forma covalente possam ser liberados, considerando as condições extremamente ácidas do meio. Nas outras etapas de purificação dos AH, como centrifugações, lavagens com água acidificada
(pH 2,0) e diálise (cinco dias), para eliminação de substâncias de baixo peso molecular e íons, os metais solúveis em água e os associados fracamente à MO (ligações eletrostáticas) vão sendo retirados, permanecendo nas amostras de $\mathrm{AH}$ apenas aqueles ligados em formas mais estáveis.

$\mathrm{O} \mathrm{Mn}$ associado à MO na forma muito estável não pode ser observado por RPE, uma vez que o íon $\mathrm{Mn}^{2+}$ apresenta uma interação fina ou separação de campo zero grande, que alarga o sinal e a linha de ressonância desaparece (Martin-Neto et al., 1988). McBride (1982) observou perda de sinal do Mn em RPE, em decorrência do grande alargamento de linha provocado por ligações muito estáveis do Mn em complexos com ácidos monocarboxílicos e policarboxílicos (AH e AF). Martin-Neto et al. (1988) também não observaram sinais de Mn em solução, extraído do solo com DTPA-TEA pH 7,3, mas intensos sinais de Mn foram observados, quando o metal foi retirado do solo com água ou pela solução Mehlich-1.

Para confirmar a hipótese de que havia Mn nas amostras de $\mathrm{AH}$, embora ele não tenha detectado por RPE, dada a sua forte associação com a MO, quantificou-se o elemento nas amostras de $\mathrm{AH}$, por espectroscopia de absorção atômica, após digestão nítrico-perclórica. Os teores foram 30,2; 25,8; 13,0 e $8,2 \mathrm{mg} \mathrm{kg}^{-1}$, respectivamente, nos locais I, II, III e IV. Nas amostras dos locais I e II, após aplicação de $48 \mathrm{~kg} \mathrm{ha}^{-1}$ de Mn, obtiveram-se 43,8 e 28,4 mg kg-1, respectivamente. Essas amostras, apesar dos maiores teores de $\mathrm{Mn}$, em relação às originais, não apresentaram sinais de Mn por RPE (Figura 4). Como discutido anteriormente, o Mn que foi liberado 
para a solução durante os processos de separação dos AH dos AF, pelo abaixamento do pH, provavelmente foi perdido durante as lavagens e diálise do material.

Dada a escassez de informações na literatura sobre teores de metais obtidos em amostras de $\mathrm{AH}$ do solo, após purificação e liofilização, torna-se difícil fazer comparações desses com outros obtidos em situações semelhantes. Senesi et al. (1989) obtiveram em amostras de $\mathrm{AH}$ de solos norteamericanos, sem aplicação de lodo de esgoto, $46 \mathrm{mg} \mathrm{kg}^{-1}$ de Mn, e Marzadori et al. (2000) quantificaram em $\mathrm{AH}$ de turfas na Itália $15 \mathrm{mg} \mathrm{kg}^{-1}$. Os teores de Mn obtidos foram inferiores aos de Senesi et al. (1989). Por sua vez, nos solos com deficiências de Mn, os teores foram superiores aos encontrados por Marzadori et al. (2000). Apesar de terem utilizado basicamente o mesmo procedimento de extração das substâncias húmicas ( $\mathrm{NaOH})$, esses autores, não utilizaram a centrifugação em alta rotação para retirada dos óxidos de ferro. Como discutido anteriormente, acredita-se que a maior purificação dos $\mathrm{AH}$ nesse estudo tenha facilitado a perda de parte do Mn adsorvido à MO e aos óxidos de ferro.

Como havia sido quantificado Mn nas amostras de $\mathrm{AH}$, extraídas dos solos dos locais I e II, o qual não pôde ser detectado por RPE (Figura 4), imaginava-se que o Mn presente nessas amostras estava associado de forma muito forte aos grupos funcionais da MO. Para comprovar essa hipótese, obtiveram-se espectros de RPE da amostra de $\mathrm{AH}$ do Local I em solução, a pH natural $(3,0)$, para verificar ou não a presença de Mn solúvel $\left[\left(\mathrm{Mn}\left(\mathrm{H}_{2} \mathrm{O}\right)_{6}\right]^{2+}\right.$, e abaixando-se o $\mathrm{pH}$ para 2,4 .
O abaixamento do $\mathrm{pH}(2,4)$ liberou o $\mathrm{Mn}$ fortemente retido aos grupos dos $\mathrm{AH}$, o qual foi visualizado por RPE, na forma de seis linhas, com interação hiperfina (A) de 90,8 Gauss (Figura 5). Desse modo, confirmaram-se as hipótese de que o Mn presente nas amostras de AH estava associado aos grupos funcionais da MO na forma de complexos de esfera interna, e que não havia Mn solúvel (solvatado), uma vez que não foram obtidos sinais a pH 3,0 (sem adição de ácido). Provavelmente, o grupo da MO envolvido na complexação do Mn seja o carboxílico, uma vez que o ele é o responsável pela complexação do metal em condições ácidas, em virtude de seu baixo valor de pKa (Manunza et al., 1995).

A partir da quantificação dos teores de Mn dos quatro solos, realizada após três meses da aplicação das doses de Mn no solo, verificou-se que, em todas as situações, as soluções ácidas $\mathrm{HCl} 0,1 \mathrm{~mol} \mathrm{~L}^{-1}$ ( $\mathrm{pH} \cong 1,1)$ e Mehlich-1 ( $\mathrm{pH} \cong 1,2)$ extraíram maiores quantidades de $\mathrm{Mn}$ do que a solução DTPA-TEA pH 7,3 (Quadro 6). As soluções ácidas têm capacidade de abaixar o pH e solubilizar o $\mathrm{Mn}$, o que não ocorre com o DTPA-TEA de reação alcalina (Galrão, 1995; Abreu \& Raij, 1996). No entanto, sabe-se que, em condições moderadamente ácidas a alcalinas, o DTPA-TEA pode quelatizar o Mn da solução, formando complexos (DTPA-Mn) de alta estabilidade (Norvell, 1991). De qualquer forma, a acidez das soluções extratoras ( $\mathrm{HCl}$ e Mehlich-1) foi mais importante na solubilização do $\mathrm{Mn}$ do que a capacidade do DTPA-TEA em quelatizar o Mn do solo.

O extrator Mehlich-3 ( $\mathrm{pH} \cong 2,4)$ extraiu maiores quantidades de Mn do que o Mehlich-1, nas parcelas que receberam a maior dose de Mn de todos os locais.

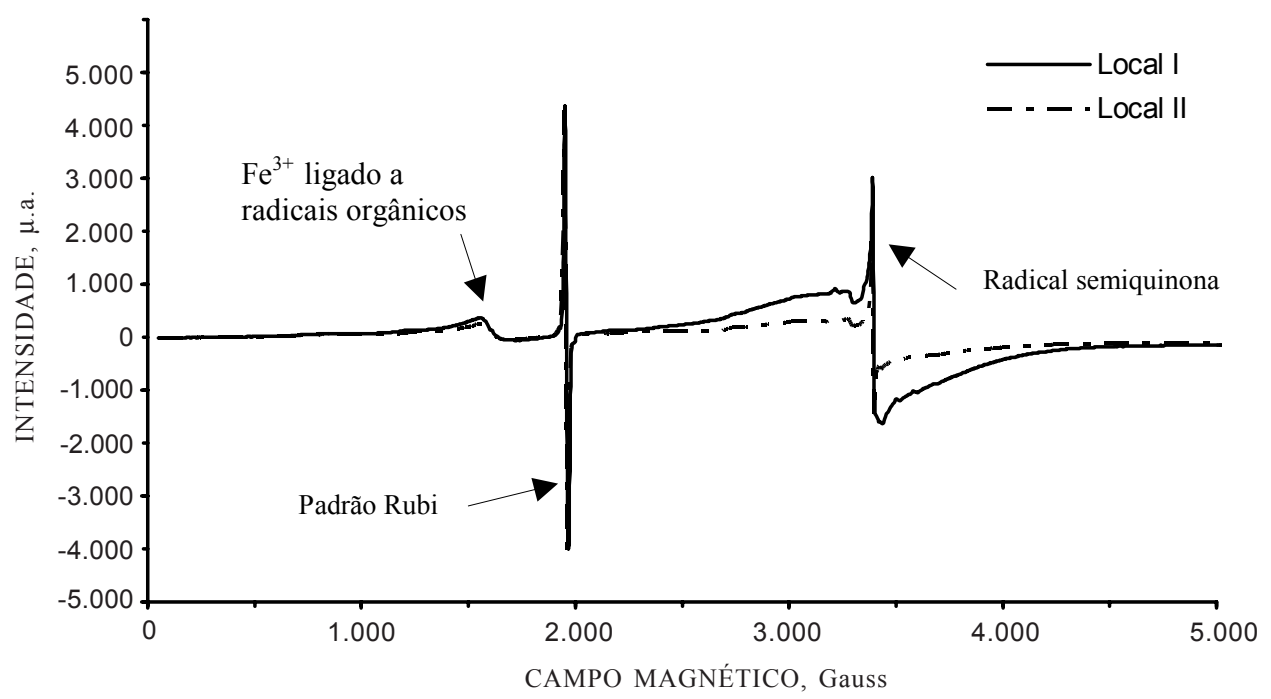

Figura 4. Espectro de RPE das amostras sólidas de AH, extraído da camada de 0,00-0,10 m, dos locais I e II, depois da centrifugação de $12.500 \mathrm{rpm}$ por $3 \mathrm{~h}$. I = intensidade. 


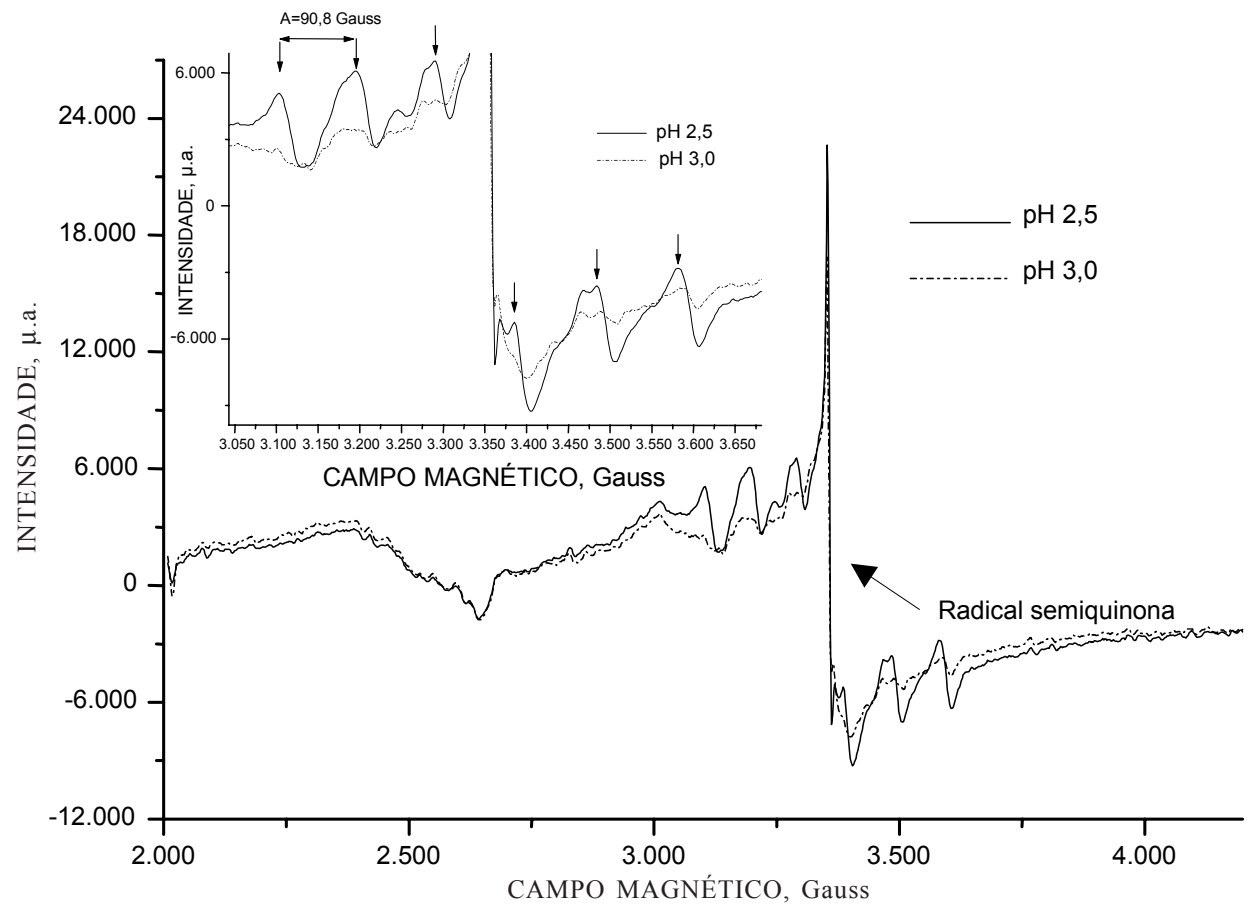

Figura 5. Espectro de RPE do AH em solução (50 mg de AH/mL de água), extraído da camada de 0,00-0,10 m do solo do Local I, a pH natural $(3,0)$ e pH 2,5 . I = intensidade.

Quadro 6. Teores de manganês na camada de 0,00-0,10 m, obtidos por diferentes extratores de acordo com a dose de manganês aplicada, em quatro locais

\begin{tabular}{|c|c|c|c|c|c|c|c|}
\hline \multirow{2}{*}{ Extrator } & \multicolumn{7}{|c|}{ Dose de manganês (kg ha-1) } \\
\hline & $\mathbf{0}$ & 1,5 & 3,0 & 6,0 & 12,0 & 24,0 & 48,0 \\
\hline & \multicolumn{7}{|c|}{$-\mathrm{mg} \mathrm{kg}^{-1}$} \\
\hline & \multicolumn{7}{|c|}{ Local $I^{(1)}$} \\
\hline $\mathrm{HCl}$ & $8,4 \mathrm{a}^{(2)}$ & $12,3 \mathrm{a}$ & $11,8 \mathrm{a}$ & $12,2 \mathrm{a}$ & $15,4 \mathrm{a}$ & $31,5 \mathrm{a}$ & $37,3 \mathrm{a}$ \\
\hline Mehlich-1 & $7,0 \mathrm{a}$ & $9,6 \mathrm{a}$ & $8,9 \mathrm{a}$ & $10,1 \mathrm{a}$ & $12,1 \mathrm{a}$ & $20,6 \mathrm{~b}$ & $23,7 \mathrm{~b}$ \\
\hline Mehlich-3 & $4,2 \mathrm{ab}$ & $7,2 \mathrm{ab}$ & $7,0 \mathrm{ab}$ & $8,3 \mathrm{a}$ & $11,1 \mathrm{a}$ & $26,5 \mathrm{ab}$ & $34,3 \mathrm{a}$ \\
\hline DTPA-TEA & $0,6 \mathrm{~b}$ & $1,2 \mathrm{~b}$ & $1,0 \mathrm{~b}$ & $1,1 \mathrm{~b}$ & $1,5 \mathrm{~b}$ & $3,1 \mathrm{c}$ & $3,2 \mathrm{c}$ \\
\hline \multirow[t]{2}{*}{ C.V. $(\%)$} & 27,4 & & & & & & \\
\hline & \multicolumn{7}{|c|}{ Local II } \\
\hline $\mathrm{HCl}$ & $16,1 \mathrm{a}$ & $19,0 \mathrm{a}$ & $19,7 \mathrm{a}$ & $18,9 \mathrm{a}$ & $21,7 \mathrm{a}$ & $28,6 \mathrm{a}$ & $41,8 \mathrm{a}$ \\
\hline Mehlich-1 & $11,3 \mathrm{ab}$ & $12,8 \mathrm{ab}$ & $12,9 \mathrm{ab}$ & $13,5 \mathrm{ab}$ & $15,7 \mathrm{ab}$ & $17,1 \mathrm{~b}$ & $24,3 \mathrm{~b}$ \\
\hline Mehlich-3 & $6,1 \mathrm{bc}$ & $7,2 \mathrm{bc}$ & $8,2 \mathrm{bc}$ & $9,0 \mathrm{bc}$ & $12,0 \mathrm{~b}$ & $16,2 \mathrm{~b}$ & $36,5 \mathrm{a}$ \\
\hline DTPA-TEA & $1,8 \mathrm{c}$ & $1,6 \mathrm{c}$ & $1,8 \mathrm{c}$ & $1,6 \mathrm{c}$ & $1,7 \mathrm{c}$ & $2,9 \mathrm{c}$ & $4,7 \mathrm{c}$ \\
\hline \multirow[t]{2}{*}{ C.V. $(\%)$} & 32,5 & $1,0 \mathrm{C}$ & $1,0 \mathrm{C}$ & $1,0 \mathrm{C}$ & $1,1 \mathrm{C}$ & $2, \mathrm{YC}^{2}$ & $4, r_{\mathrm{C}}$ \\
\hline & \multicolumn{7}{|c|}{ Local III } \\
\hline $\mathrm{HCl}$ & $15,5 \mathrm{a}$ & $14,6 \mathrm{a}$ & $15,2 \mathrm{a}$ & $17,4 \mathrm{a}$ & $18,6 \mathrm{a}$ & $21,3 \mathrm{a}$ & $29,1 \mathrm{a}$ \\
\hline Mehlich-1 & $14,6 \mathrm{a}$ & $14,5 \mathrm{a}$ & $14,5 \mathrm{a}$ & $16,4 \mathrm{a}$ & $17,2 \mathrm{a}$ & $18,2 \mathrm{ab}$ & $23,7 \mathrm{~b}$ \\
\hline Mehlich-3 & $7,8 \mathrm{~b}$ & $8,1 \mathrm{~b}$ & $8,8 \mathrm{~b}$ & $11,1 \mathrm{~b}$ & $12,5 \mathrm{~b}$ & $16,8 \mathrm{~b}$ & $28,1 \mathrm{a}$ \\
\hline DTPA-TEA & $5,0 \mathrm{~b}$ & $4,8 \mathrm{~b}$ & $4,7 \mathrm{c}$ & $5,6 \mathrm{c}$ & $5,9 \mathrm{c}$ & $8,1 \mathrm{c}$ & $12,1 \mathrm{c}$ \\
\hline \multirow[t]{2}{*}{ C.V. $(\%)$} & 14,4 & & & & & & \\
\hline & \multicolumn{7}{|c|}{ Local IV } \\
\hline $\mathrm{HCl}$ & $30,1 \mathrm{a}$ & $29,8 \mathrm{a}$ & $29,2 \mathrm{a}$ & $30,8 \mathrm{a}$ & $45,6 \mathrm{a}$ & $35,1 \mathrm{a}$ & $49,8 \mathrm{a}$ \\
\hline Mehlich-1 & $14,8 \mathrm{~b}$ & $14,3 \mathrm{~b}$ & $15,2 \mathrm{~b}$ & $15,1 \mathrm{~b}$ & $17,8 \mathrm{~b}$ & $17,7 \mathrm{~b}$ & $19,9 \mathrm{c}$ \\
\hline Mehlich-3 & $10,3 \mathrm{bc}$ & $11,2 \mathrm{bc}$ & $10,0 \mathrm{bc}$ & $12,0 \mathrm{bc}$ & $19,8 \mathrm{~b}$ & $16,2 \mathrm{~b}$ & $28,3 \mathrm{~b}$ \\
\hline DTPA-TEA & $6,8 \mathrm{c}$ & $6,4 \mathrm{c}$ & $6,4 \mathrm{c}$ & $6,8 \mathrm{c}$ & $9,8 \mathrm{c}$ & $8,8 \mathrm{c}$ & $12,1 \mathrm{~d}$ \\
\hline C.V. (\%) & 18,9 & & & & & & \\
\hline
\end{tabular}


Apesar de o EDTA, presente no Mehlich-3, ser mais eficiente na complexação do Mn em condições alcalinas do que em condições ácidas (Norvell, 1991), nesse caso, sua capacidade de quelação, aliada à acidez $(\mathrm{pH} \cong 2,4)$, foi mais importante do que a maior acidez do Mehlich-1 ( $\mathrm{pH} \cong 1,2)$. Mehlich (1984) também observou em solos norte-americanos que o Mehlich-3 extraiu maiores quantidades de Mn do que o Mehlich-2, o qual não continha EDTA em sua composição.

Em muitos casos, o Mehlich-3 (pH $\cong 2,4)$ extraiu maiores teores do que o DTPA-TEA pH 7,3, o que não era esperado, uma vez que o quelato DTPA-Mn tem alta estabilidade em condições de elevado $\mathrm{pH}$ e o complexo EDTA-Mn é mais estável em condições moderadamente ácidas a alcalinas (Tisdale et al., 1985; Norvell, 1991). Além disso, a concentração do DTPA-TEA $\left(0,005 \mathrm{~mol} \mathrm{~L}^{-1}\right)$ na solução extratora é superior à do EDTA na solução Mehlich-3 $\left(0,001 \mathrm{~mol} \mathrm{~L}^{-1}\right)$. Por outro lado, as soluções ácidas têm capacidade de abaixar o $\mathrm{pH}$ e solubilizar o $\mathrm{Mn}$, o que não ocorre com o DTPA-TEA de reação alcalina (Galrão, 1995; Abreu \& Raij, 1996). Assim, a acidez da solução extratora prevaleceu na solubilização do Mn sobre a capacidade de quelação do DTPA-TEA.

Com exceção dos teores de Mn, extraídos por DTPA-TEA, nas amostras de terra dos locais I e II, os teores de Mn aumentaram com as doses
(Figura 6), o que era esperado em razão das elevadas quantidades aplicadas. Miyazawa et al. (1996) e Silva (2000) também observaram aumento dos teores de Mn, com aplicação do nutriente no solo. No entanto, nos estudos de Miyazawa et al. (1996), após o sétimo mês de avaliação, os teores de Mn dos tratamentos que receberam as menores doses (abaixo de $100 \mathrm{mg} \mathrm{kg}^{-1}$ ) foram reduzidos para teor igual ao do tratamento testemunha. As diminuições dos teores de Mn foram atribuídas apenas às reações de oxidação e redução, das quais o Mn participa como carreador de elétrons (Borkert et al., 2001), descartando-se a possibilidade de formação de compostos estáveis do Mn com a MO, que pode ser mais importante em solos sob SSD (Tisdale et al., 1985; Sims, 1986), resultante dos maiores teores de MO.

A concentração e a quantidade de Mn acumuladas pelas plantas de soja variaram muito pouco com as doses (Figura 7), havendo uma pequena elevação somente nos locais I e III. Em solos sob cerrado, Silva (2000) também não obteve aumento da concentração de Mn nas folhas de soja com a aplicação de $4 \mathrm{~kg} \mathrm{ha}^{-1}$ de Mn, nas formas de sulfato e oxissulfato, em experimentos de casa de vegetação e campo.

Houve grande diferença entre o acúmulo de Mn do Local I com relação aos demais, em decorrência de menor produção de matéria seca. Além disso, as
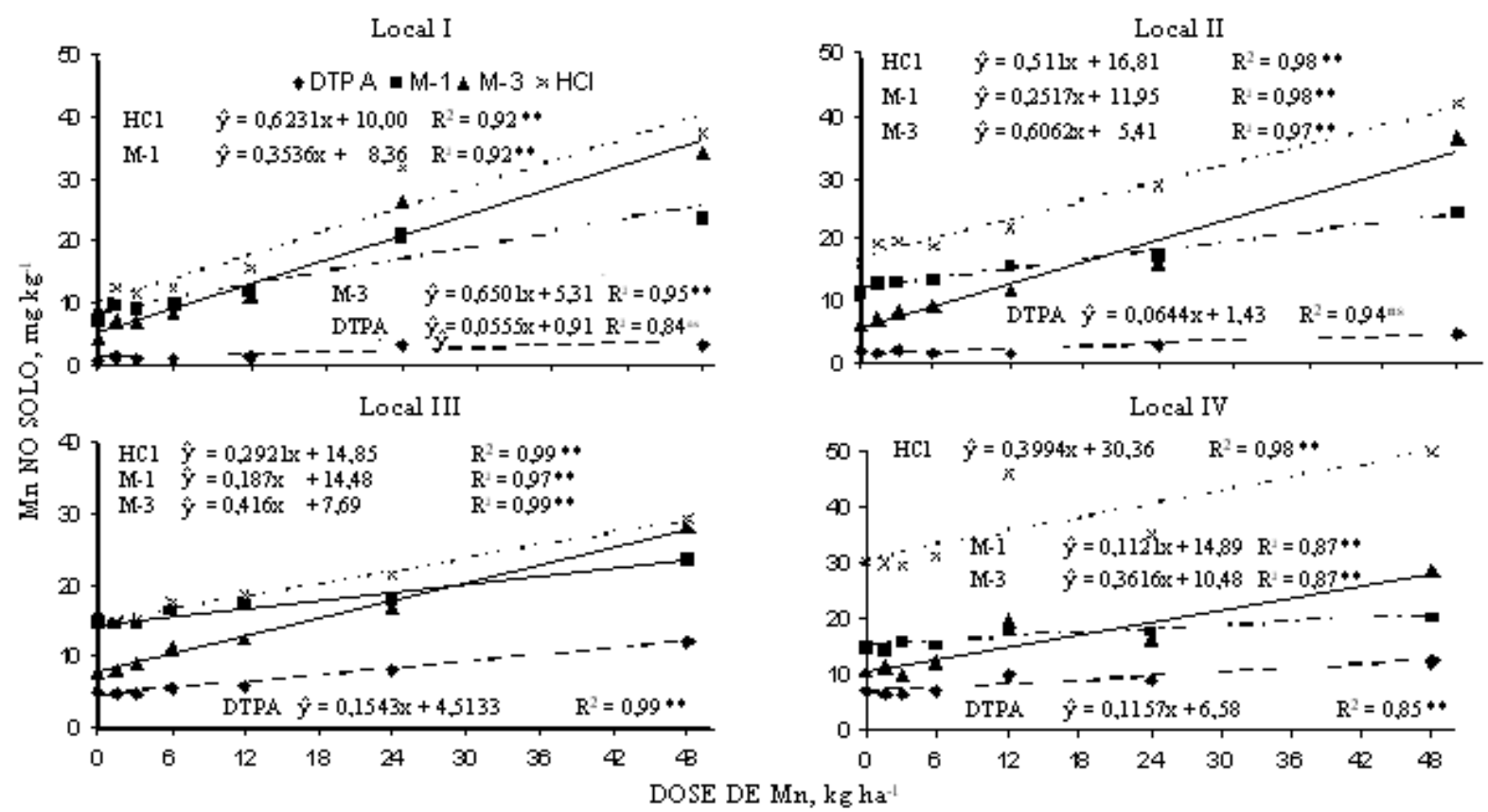

Figura 6. 'Teores de manganês do solo, extraídos com diferentes extratores (D'TPA-'TEA pH 7,3, M-1 = Mehlich1, M-3 = Mehlich-3 e HCl 0,1 mol L ${ }^{-1}$ ), dos quatro locais, considerando as doses de manganês aplicadas. Local I: Faz. Santa Rosa; Locais II e III: Faz. Novo Horizonte; Local IV: Campo Experimental da Fundação ABC. Locais I e II: já apresentaram deficiências de manganês em soja; Locais III e IV: sem registros de deficiências. Os locais I, III, IV estão localizados no município de Tibagi, e o Local IV, em Castro (PR). ns = não-significativo $\mathrm{e}^{* *}$ significativo a $1 \%$. 

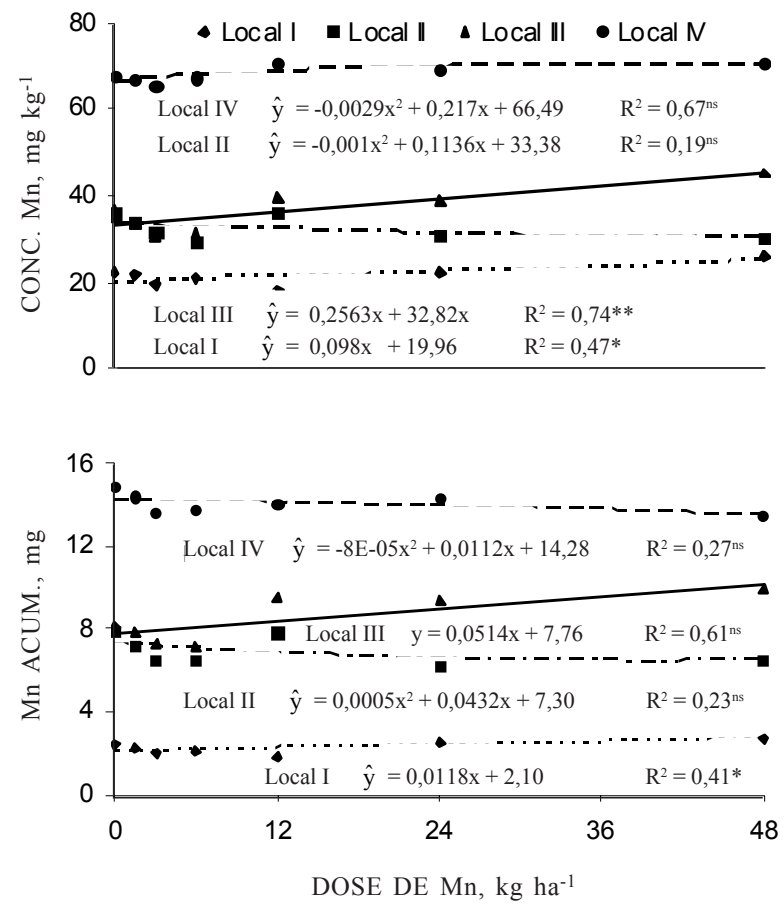

Figura 7. Concentração e acúmulo de manganês na parte aérea das plantas de soja, nos quatro locais, considerando as doses de manganês aplicadas. Local I: Faz. Santa Rosa; Locais II e III: Faz. Novo Horizonte; Local IV: Campo Experimental da Fundação ABC. Locais I e II: já apresentaram deficiências de manganês em soja; Locais III e IV: sem registros de deficiências. Os locais I, III, IV estão localizados no município de Tibagi, e o Local IV, em Castro (PR). ns = nãosignificativo, * e ** significativos a 5 e $1 \%$.

plantas cultivadas nesse local apresentaram menor concentração de Mn na parte aérea, comparada à de outros locais. A menor produção de matéria seca do Local I em relação aos demais ocorreu devido ao atraso da semeadura e germinação da soja, causado por um veranico em Tibagi, PR (19/10 a 09/11/2001).

Como a concentração e o acúmulo de Mn na parte aérea das plantas praticamente não variaram com as doses, acredita-se que grande parte do nutriente aplicado foi complexada pela MO, como discutido anteriormente. Parte do Mn também foi adsorvida pelos óxidos, como mostrado na figura 1.

Provavelmente, as deficiências de Mn em soja cultivada sob SSD, ocorridas em solos com teores considerados adequados, acontecem porque grande parte do Mn do solo, extraído pelos diversos extratores, não está disponível às plantas, mas associado à MO. Prova disso é que vêm sendo observadas correlações diretas entre os teores de Mn do solo e de MO (Castro et al., 1992; Edwards et al., 1992; Motta et al., 2002) e correlações inversas entre o teor de Mn da fração orgânica e a quantidade de Mn absorvida pelas plantas (Sims, 1986).

No presente estudo, as correlações entre a concentração e o acúmulo de Mn com os teores das formas trocáveis e orgânicas foram baixas e não significativas $(p>0,05)$, considerando o pequeno número de dados (12 parcelas $=$ três doses $\mathrm{x}$ quatro blocos). No entanto, verificou-se que os teores de Mn do solo aumentaram com as doses aplicadas (Figura 6) e que grande parte do Mn aplicado foi retido pela MO (Figura 1) mostrando que os teores extraídos por esses extratores podem não ser disponíveis.

Os coeficientes de correlação linear simples (r) entre teores de Mn no solo, extraídos pelos diversos extratores, e concentração e quantidade de Mn acumuladas na parte aérea, geralmente foram baixos, havendo valores de $\mathrm{r}$ significativos apenas nos locais I e III (Quadro 7). No Local I, com exceção do Mehlich-1, os coeficientes de correlação entre a concentração de Mn na parte aérea e os teores no solo foram significativos. No caso do Local III, com exceção do valor de $\mathrm{r}$ entre os teores extraídos com Mehlich-1 e a quantidade acumulada na parte aérea, os valores de $\mathrm{r}$ foram significativos.

Os baixos coeficientes de correlação obtidos entre os teores de Mn no solo e a concentração e a quantidade acumuladas na parte aérea eram esperados, uma vez que os atributos da planta praticamente não variaram com as doses aplicadas em cada local. Como havia diferenças entre os locais na concentração e na quantidade acumulada de Mn e nos teores de Mn no solo, procederam-se estudos de correlação por meio das variáveis de todos os locais em conjunto. Nesse caso, a amplitude entre os atributos da planta foi aumentada e maiores valores de $\mathrm{r}$ foram obtidos entre os teores de Mn no solo, extraídos com DTPA-TEA, e a concentração e quantidade acumuladas pelas plantas (Figuras 7 e 8).

Resultados similares, quanto à eficiência do DTPA-TEA, foram observados por outros autores em solos que receberam aplicação de lodo de esgoto, em condições norte-americanas (Mulchi et al., 1987; 1991; Brown et al., 1997), e em alguns estudos desenvolvidos no estado de São Paulo, em solos sem aplicação de lodo (Camargo et al., 1982; Rosolem et al., 1992).

Em experimento desenvolvido em casa de vegetação, com aplicação de doses de Mn, Abreu et al. (1996) obtiveram maior coeficiente de determinação $\left(\mathrm{R}^{2}=0,76\right)$ entre o $\mathrm{Mn}$ absorvido pela soja e o $\mathrm{Mn}$ extraído do solo por DTPA-TEA, comparado ao Mehlich-1 $\left(\mathrm{R}^{2}=0,67\right)$. Em outro trabalho, realizado por Borkert et al. (1984), a solução de $\mathrm{HCl}$ foi a mais ineficiente na avaliação da disponibilidade de Mn entre os diversos extratores avaliados $\left(\mathrm{SrCl}_{2} 1 \mathrm{~mol} \mathrm{~L}^{-1}\right.$; $\mathrm{NH}_{4} \mathrm{OAc} 1 \mathrm{~mol} \mathrm{~L}^{-1} \mathrm{pH}$ 7,0; $\mathrm{Mg}\left(\mathrm{NO}_{3}\right)_{2}$ 0,5 $\mathrm{mol} \mathrm{L}^{-1} \mathrm{e}$

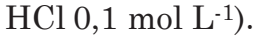


Quadro 7. Coeficientes de correlação linear (r) entre teores de manganês no solo, extraídos por diversos extratores, e concentração de manganês e quantidade de manganês acumuladas na parte aérea, nos quatro locais estudados

\begin{tabular}{|c|c|c|c|c|}
\hline Atributo & DTPA-TEA & Mehlich-1 & Mehlich-3 & $\mathrm{HCl}$ \\
\hline & \multicolumn{4}{|c|}{ Local I(1) } \\
\hline Concentração na parte aérea & $0,42^{*}$ & $0,35 \mathrm{~ns}$ & $0,39^{*}$ & $0,41^{*}$ \\
\hline \multirow[t]{2}{*}{ Acumulado na parte aérea } & $0,36 \mathrm{~ns}$ & $0,26 \mathrm{~ns}$ & $0,32 \mathrm{~ns}$ & $0,33 \mathrm{~ns}$ \\
\hline & \multicolumn{4}{|c|}{ Local II } \\
\hline Concentração na parte aérea & $-0,19 \mathrm{~ns}$ & $-0,15 \mathrm{~ns}$ & $-0,19 \mathrm{~ns}$ & $-0,21 \mathrm{~ns}$ \\
\hline \multirow{2}{*}{ Acumulado na parte aérea } & $-0,16 \mathrm{~ns}$ & $-0,07 \mathrm{~ns}$ & $-0,15 \mathrm{~ns}$ & $-0,18 \mathrm{~ns}$ \\
\hline & \multicolumn{4}{|c|}{ Local III } \\
\hline Concentração na parte aérea & $0,45^{*}$ & $0,45^{*}$ & $0,50^{*}$ & $0,45^{*}$ \\
\hline \multirow[t]{2}{*}{ Acumulado na parte aérea } & $0,44^{*}$ & $0,34 \mathrm{~ns}$ & $0,45^{*}$ & $0,37^{*}$ \\
\hline & \multicolumn{4}{|c|}{ Local IV } \\
\hline Concentração na parte aérea & $0,17 \mathrm{~ns}$ & $0,21 \mathrm{~ns}$ & $0,16 \mathrm{~ns}$ & $0,23 \mathrm{~ns}$ \\
\hline Acumulado na parte aérea & $0,09 \mathrm{~ns}$ & $0,10 \mathrm{~ns}$ & $0,01 \mathrm{~ns}$ & $0,01 \mathrm{~ns}$ \\
\hline
\end{tabular}

(1) Local I: Fazenda Santa Rosa; Locais II e III: Fazenda Novo Horizonte; Local IV: Campo Experimental da Fundação ABC. Os locais I, III, IV estão localizados no município de Tibagi, e o Local IV, em Castro (PR). = não-significativo e * significativo a $5 \%$.

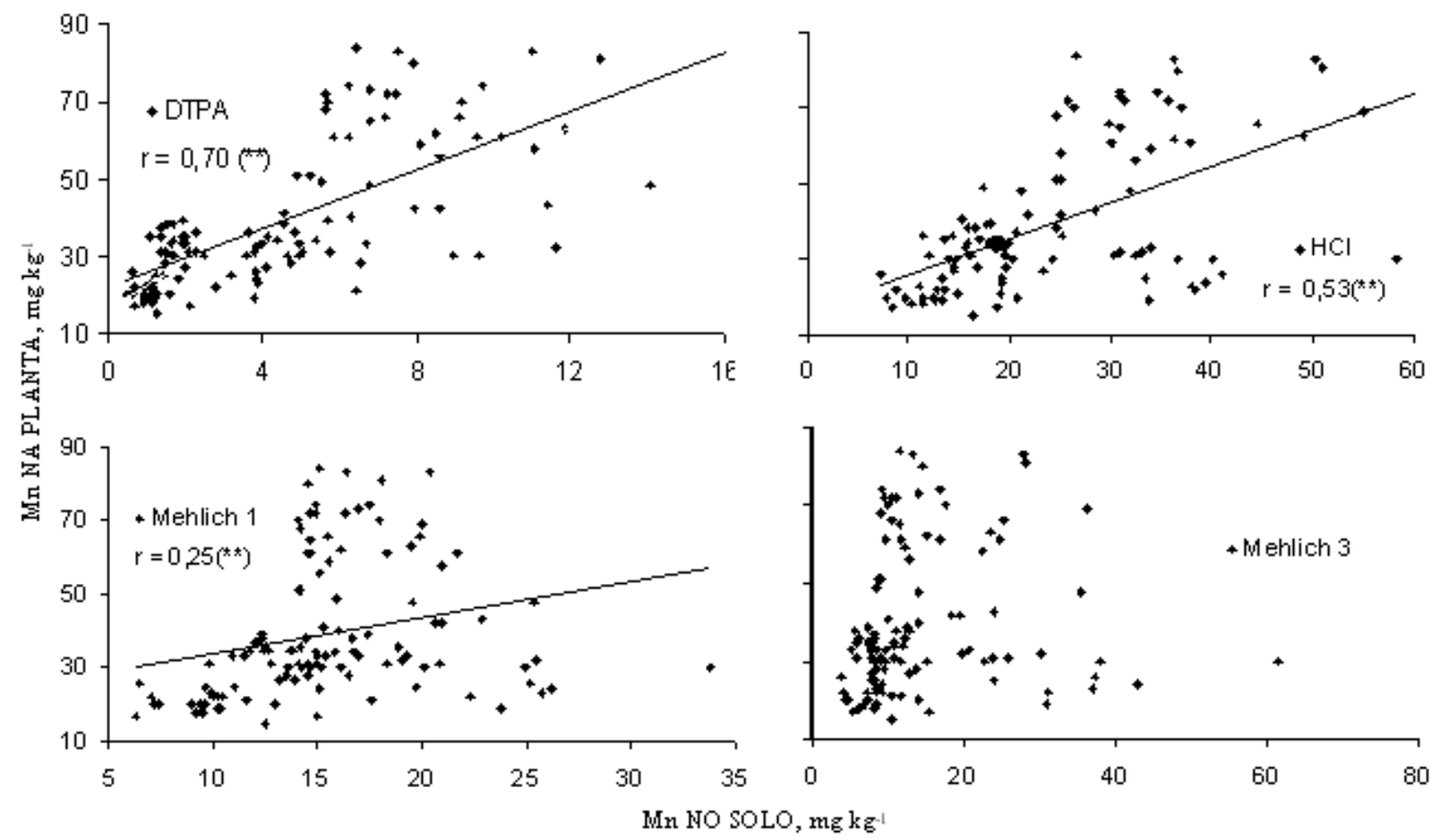

Figura 8. Correlações entre os teores de manganês do solo, extraídos por diversos extratores, após três meses da aplicação ao solo com a concentração de manganês na parte aérea. ns = não-significativo, * $\mathrm{e}^{* *}$ significativo a 1 e $5 \%$.

As produtividades de soja não variaram com as doses em nenhum dos locais (Figura 9). A complexação de grande parte do Mn aplicado pela MO e o aumento do Mn na forma de óxidos no solo
(Figura 1) podem explicar a ausência de resposta na produtividade de soja observada neste trabalho e por outros autores (Pauletti, 1998; Silva, 2000) em solos sob SSD, deficientes do nutriente. 

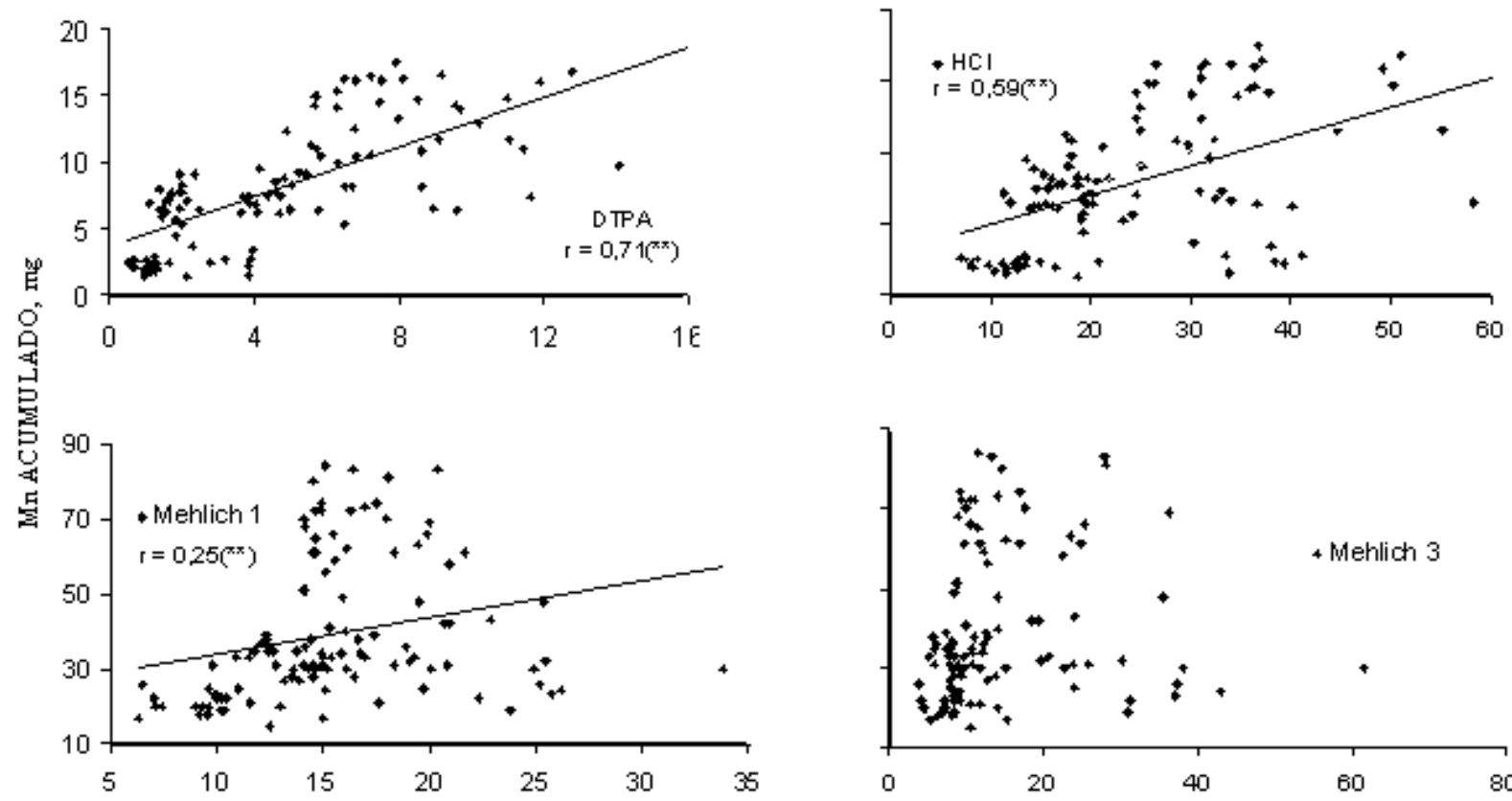

$\mathrm{Mn}$ HO SOLO, mg k

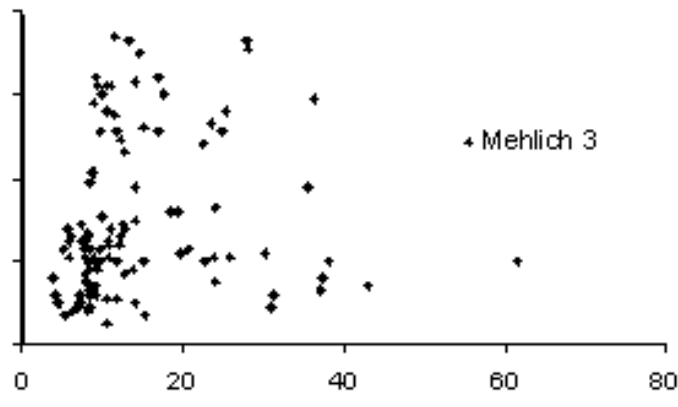

F'igura 9. Correlaçōes entre os teores de manganès do solo, extraidos por diversos extratores, após très meses da aplicação ao solo com a quantidade de manganês acumulado na parte aérea. ns $=$ nãosignificativo, * e ** significativo a 1 e $5 \%$.

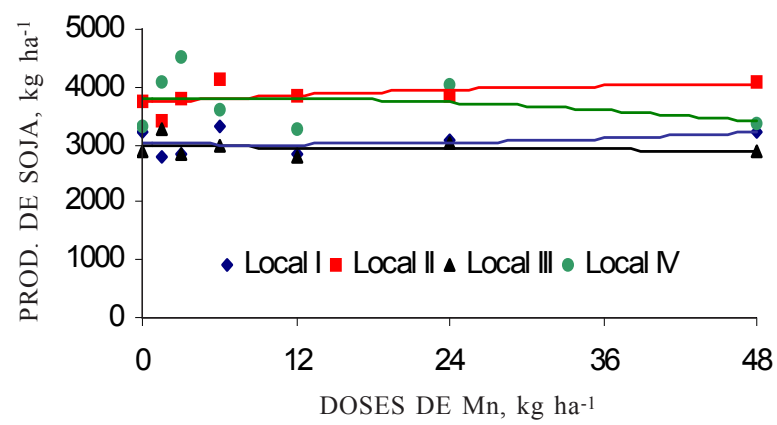

Figura 10. Produtividade de soja em quatro locais, considerando as doses de manganês. Local I: Fazenda Santa Rosa; Locais II e III: Fazenda Novo Horizonte; Local IV: Campo Experimental da Fundação ABC. Locais I e II: com constatações de deficiências de manganês em soja; Locais III e IV: sem registros de deficiências. Os locais I, III, IV estão localizados no município de Tibagi, e o Local IV, em Castro (PR).

Como as produtividades de soja não variaram com doses de até $48 \mathrm{~kg} \mathrm{ha}^{-1}$ de $\mathrm{Mn}$, acredita-se que as doses aplicadas não tenham sido suficientes, graças à complexação de grande parte do nutriente pela MO. Assim, novos estudos devem ser realizados em condições de campo em solos sob SSD com altos teores de MO, utilizando doses mais elevadas. É provável que as doses atualmente recomendadas (14-
$17 \mathrm{~kg} \mathrm{ha}^{-1}$ a lanço, e $3-4 \mathrm{~kg} \mathrm{ha}^{-1}$, no sulco de semeadura) (Borkert et al., 2001) sejam baixas para esses solos.

\section{CONCLUSÕES}

1. Com base nos resultados obtidos pela extração seqüencial e pela ressonância paramagnética eletrônica, concluiu-se que grande parte do Mn aplicado nos solos sob semeadura direta é retida na fração orgânica em formas estáveis, não-disponíveis às plantas.

2. Os teores de Mn trocáveis extraídos pelos di-

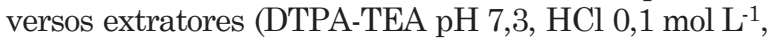
Mehlich-1 e 3) geralmente não representaram a quantidade disponível às plantas. De todos os extratores, o DTPA-TEA foi o mais adequado na avaliação da disponibilidade de Mn à soja.

\section{LITERATURA CITADA}

ABREU, C.A. \& RAIJ, B. van. Efeito da reação do solo no zinco extraído pelas soluções de DTPA-TEA e Mehlich-1. Bragantia, 55:357-363, 1996.

AHNSTROM, Z.S. \& PARKER, D.R. Development and assessment of a sequential extraction procedure for the fractionation of soil cadmium. Soil Sci. Soc. Am. J., 63:1650-1658, 1999 . 
ALEXANDROVA, L.N. The use of sodium pyrophosphate for isolating free humic substances and their organic mineral compounds form soil. Soviet Soil Sci., 2:190-197, 1960.

BAYER, C.; DICK, D.P.; RIBEIRO, G.M. \& SCHEUERMANN, K.K. Carbon stocks in organic matter fractions as affected by land use and soil management, with emphasis on notillage effect. Ci. Rural, 32:401-406, 2002a.

BAYER, C.; MARTIN-NETO, L.; MIELNICZUKC, J. \& ERNANI, P.R. Tillage and cropping system effects on soil humic acid characteristic as determined by electron spin resonance and fluorescence spectroscopies. Geoderma, 105:81-92, 2002b.

BIBAK, A. Cobalt, copper, and manganese adsorption by aluminium and iron oxides and humic acid. Comm. Soil Sci. Plant Anal., 25:3229-3239, 1994.

BORKERT, C.M.; LANTMANN, A.F; PALHANO, J.B. \& SFREDO, G.J. Determinação química do manganês absorvível pela soja. In: SEMINÁRIO NACIONAL DE PESQUISA DA SOJA, 3., Campinas, 1984. Anais. Londrina, Embrapa/CNPSo, 1984. p.879-887.

BORKERT, C.M.; PAVAN, M.A. \& BATAGLIA, O.C. Disponibilidade e avaliação de elementos catiônicos: ferro e manganês. In: FERREIRA, M.E.; CRUZ, M.C.P.; RAIJ, B. van \& ABREU, C.A., eds. Micronutrientes elementos tóxicos na agricultura. Jaboticabal: CNPq/FAPESP/ POTAFOS, 2001. p.151-186.

BOWMAN, R.A. \& HALVORSON, A.D. Soil Chemical after nine years of differential $\mathrm{N}$ fertilization in a no-till dryland wheat-corn-fallow rotation. Soil Sci., 163:241-247, 1998.

BROWN, S.; ANGLE, J.S. \& CHANEY, R.L. Correction of limed biosolid induced manganese deficiency on a longterm field experiment. J. Environ. Qual., 26:1375-1384, 1997.

CAIRES, E.F.; BANZATTO, D.A. \& FONSECA, A.F. Calagem na superfície em sistema de plantio direto. R. Bras. Ci. Solo, 24:161-170, 2000.

CAIRES, E.F.; FONSECA, A.F.; MENDES, J.; CHUEIRI, W.A. \& MADRUGA, E.F. Produção de milho, trigo e soja em função das alterações das características químicas do solo pela aplicação de calcário e gesso na superfície, em sistema de plantio direto. R. Bras. Ci. Solo, 23:315-327, 1999.

CAMARGO, O.A.; VALADARES, J.M.A.S. \& DECHEN, A.R. Efeitos do $\mathrm{pH}$ e da incubação na extração do manganês, zinco, cobre e ferro do solo. R. Bras. Ci. Solo, 6:83-88, 1982 .

CANELLAS, L.P.; SANTOS, G.A. \& AMARAL SOBRINHO, N.M.B. Reações da matéria orgânica. In: SANTOS, G.A. \& CAMARGO, F.A.O., ed. Fundamentos da matéria orgânica do solo: ecossistemas tropicais e subtropicais. Porto Alegre, Genesis, 1999. p.69-90.

CASTRO, O.M.; CAMARGO, O.A.; CANTARELLA, H.; VIEIRA, S.R. \& DECHEN, S.C.F. Teores de zinco, cobre, manganês e ferro em dois Latossolos sob plantio direto e convencional. Bragantia, 51:77-84, 1992.
CERETTA, C.A.; BAYER, C.; DICK, D.P.; MARTIN-NETO, L. \& COLNAGO, L.A. Métodos espectroscópicos. In: SANTOS, G.A. \& CAMARGO, F.A.O., eds. Fundamentos da matéria orgânica do solo: ecossistemas tropicais e subtropicais. Porto Alegre, Genesis, 1999. p.293-336.

CHANG, A.C.; PAGE, A.L.; WARNEKE, J.E. \& GRGUREVIC, E. Sequential extraction on soil heavy metals following a sludge application. J. Environ. Qual., 13:33-38, 1984.

CHAO, T.T. Use of partial dissolutin techniques in geochemical exploration. J. Geochem. Exploration, 20:101-135, 1984.

EDWARDS, J.H.; WOOD, C.W.; THURLOW, D.L. \& RUF, M.E. Tillage and crop rotation on fertility status of a Hapludult soil. Soil Sci. Soc. Am. J., 56:1577-1582, 1992.

EMPRESA BRASILEIRA DE PESQUISA AGROPECUÁRIA EMBRAPA. Serviço nacional de levantamento e conservação de solos. Manual de métodos de análise de solo. Método 2-6: análise química: fósforo assimilável. Rio de Janeiro, 1979. 271p.

EVANGELOU, V.P. \& MARSI, M. Composition and metal ion complexation behavour of humic factions derived from corn tissue. Plant Soil, 229:13-24, 2001.

FOLLETT, R.F. \& LINDSAY, W.L. Changes in DTPAextractable Zinc, iron, manganese, and coper in soils followiing fertilization. Soil Sci. Soc. Am. J., 35:600-602, 1971.

FOLLETT, R.F. \& PETERSON, G.A. Surface soil nutrient distribution as affected by wheat- fallow tillage systems. Soil Sci. Soc. Am. J., 52:141-147, 1988.

GALRÃO, E.Z. Níveis críticos de Zn para o milho cultivado em Latossolo Vermelho-Amarelho, fase cerrado. R. Bras. Ci. Solo, 19:255-260, 1995.

GROVE, J.H. \& BLEVINS, R.L. Correcting soil acidification in continuous corn (Zea Mays L.). Comm. Soil Sci. Plant Anal., 19:1331-1342, 1988.

JUO, A.S.R.; DABIRI, A. \& FRANZLUEBBERS, A.J. Acidification of kaolinitic alfisol under cropping with nitrogen fertilization in West Africa. Plant Soil, 17:245253, 1995.

LAKATOS, B.; TIBAI, T. \& MEISEL, J. EPR espectra of humic acids and their metal complexes. Geoderma, 19:319-338, 1977.

LINDSAY, W.L \& NORVELL, W.A. Development of a DTPA soil test for zinc, iron, manganese, and copper. Soil Sci. Soc. Am. J, 42:421-428, 1978.

MALAVOLTA, E.; VITTI, G.C. \& OLIVEIRA, S.A. Avaliação do estado nutricional das plantas: princípios e aplicações. Piracicaba-SP: POTAFOS, 1997. 319p.

MANUNZA, B.; DEIANA, S.; MADDAU, V.; GESSA, C. \& SEEBER, R. Stability constants of metal-humate complexes: titration data analyzed by bimodal gaussian distribution. Soil Sci. Soc. Am. J., 59:1570-1574, 1995. 
MARTIN-NETO, L.; BISCEGLI, C.I. \& COLNAGO, L.A. Ressonância paramagnética nuclear: aplicações em solo. In: ESCUELA LATINO-AMERICANA DE FÍSICA DE SUELOS, 1., São Carlos, 1988. Anais. São Carlos, Embrapa/CNPDIA, 1988. paginação irregular. ref.I ELAFS/88-27B.

MARZADORI, C.; FRANCIOSO, O.; CIAVATTA, C. \& GESSA, C. Activity and stability of jack bean urease in the presence of peat humic acids obtained using different extractants. Biol. Fert. Soils, 32:415-420, 2000.

McBRIDE, M.B. Electron Spin Resonance investigation of $\mathrm{Mn}^{2+}$ complexation in natural and synthetic organics. Soil Sci. Soc. Am. J., 46:1137-1143, 1982.

MEHLICH, A. Mehlich-3 soil test extractant: a modification of Mehlich-2 extractant. Comm. Soil Sci. Plant Anal. 15:1409$1416,1984$.

MIYAZAWA, M.; PAVAN, M.A.; MACHADO, P.L.O.A.; OLIVEIRA, E.D. \& YAMASCHITA, M. Manganese dynamic in acid soils and uptake by maize seedlings. Comm. Soil Sci. Plant Anal., 27:2347-2359, 1996.

MOREIRA, S.G. Formas químicas no solo e disponibilidade de micronutrientes à soja em sistema de semeadura direta. Piracicaba, Escola Superior de Agricultura "Luiz de Queiroz", 2003, 147p. (Tese Doutorado)

MOREIRA, S.G.; KIEHL, J.C.; PROCHNOW, L.I. \& PAULETTI, V. Calagem em sistema de semeadura direta e efeitos sobre a acidez do solo, disponibilidade de nutrientes e produtividade de milho e soja. R. Bras. Ci. Solo, 25:71-81, 2001.

MOTTA, A.C. \& PREVEDELLO, B.M.S. Micronutrientes: fatores que afetam a disponibilidade e respostas das culturas. In: SÁ, J.C.M. coord. Curso sobre o manejo do solo no sistema de plantio direto. Castro, Fundação ABC, 1995. p.302-327.

MOTTA, A.C.; REEVES, D.W. \& TOUCHTON; J.T. Tillage intensity effects on chemical indicators of soil quality in two coastal plain soils. Comm. Soil Sci. Plant Anal., 33:913932, 2002.

MULCHI, C.L.; ADAMU, C.A.; BELL, P.F. \& CHANEY, R.L. Residual heavy metal concentrations in sludgeamended coastal plain soils. I. Comparison of extractants. Comm. Soil Sci. Plant Anal., 22:919-941, 1991.

MULCHI, C.L.; BELL, P.F.; ADAMU, C. \& CHANEY,R. Long term availability of metals in sludge amended acid soils. J. Plant Nutr., 10:1149-1161, 1987.

NASCIMENTO, C.W.A.; FONTES, R.L.F. \& NEVES, J.C.L. Dessorção, extração e fracionamento de Manganês em Latossolos. R. Bras. Ci. Solo, 26:589-597, 2002.

NORVELL, W.A. Reactions of metal chelates in soils and nutrient solutions. In: MORTVEDT, J. J.; COX, F.R.; SHUMAN, L.M. \& WELCH, R.M., eds. Micronutrients in agriculture. Madison, Soil Science Society of America, 1991. p.187-228.

NOVOTNY, E.H. Estudos espectroscópicos e cromatográficos de substâncias húmicas do solo sob diferentes sistemas de preparo. São Carlos, Instituto de Química de São Carlos, Universidade de São Paulo, 2002. 215p. (Tese de Doutorado)
PAULETTI, V. Disponibilidade e resposta de culturas a micronutrientes em solos arenosos. In: FRIES, M.R., coord. Plantio direto em solos arenosos: alternativas de manejo para a sustentabilidade agropecuária. Santa Maria, Universidade Federal de Santa Maria, 1998. p.82-103.

RAIJ, B. van; QUAGGIO, J.A.; CANTARELLA, H.; FERREIRA, M.E.; LOPES, A.S. \& BATAGLIA, O.C. Análise química do solo para fins de fertilidade. Campinas, Fundação Cargill, 1987. 170p.

ROSOLEM, C.A.; BESSA, M.A.; AMARAL, P.G. \& PEREIRA, H.F.M. Manganês no solo, sua avaliação e toxidez de manganês em soja. Pesq. Agropec. Bras., 27:277-285, 1992.

SAAB, S.C. Caracterização da matéria orgânica em gleissolos por espectroscopias de EPR, RMN, IV e UV-visível. São Carlos, Universidade de São Paulo, 1999. 111p. (Tese de Doutorado)

SANZONOWICZ, C. Deficiência de manganês em solos do cerrado. POTAFOS, 1995. $7 \mathrm{p}$.

SCHNITZER, M. Organic matter characterization. In: PAGE, A.L., ed. Methods of soil analysis. Madison, America Society of Agronomy, 1982. p.581-594.

SCHNITZER, M. \& KHAN, S.U. Soil organic matter. Amsterdam, Elsevier, 1978. 319p.

SENESI, N. Application of electron spin resonance (ESR) spectroscopy in soil chemistry. Soil Adv. Sci., 14:77-123, 1990.

SENESI, N. \& SPOSITO, G. Characterization and stability of transition metal complexos of chestnut (Castanea sativa L.) leaf litter. J. Soil Sci., 40:461-472, 1989.

SENESI, N.; SPOSITO, G.; HOLTZCLAW, K.M. \& BRADFORD, G.R. Chemical properties of metal-humic acid fractions of a sewage sludge-amended aridisol. J. Environ. Qual., 18:186-194, 1989.

SHUMAN, L.M. Fractionation method for soil micronutrients. Soil Sci.,140:11-16, 1985.

SILVA, M.M. Fontes e modos de aplicação de manganês na nutrição, produção e qualidade da soja, cultivada em solo de cerrado. Piracicaba, Escola Superior de Agricultura "Luiz de Queiroz", 2000. 174p. (Tese de Doutorado)

SIMS, J.T. Soil pH effects on the distribution and plant availability of manganese, copper, and zinc organic matter in soil fractions. Soil Sci. Soc. Am. J., 50:367-373, 1986.

STEVENSON, F.J. Humus chemistry: Genesis, composition, reactions. New York, John Wiley \& Sons, 1994. 496p.

TANAKA, R.T.; MASCARENHAS, H.A.A. \& BULISANI, E.A. Manganese deficiency in soybean induced by excess lime. Better Crops Inter., 9:7, 1993.

TISDALE, S.L.; NELSON, W.L. \& BEATON, J.D. Soil fertility and fertilizers. New York, 1985. 754p.

VALADARES, J.M.A.S. \& CAMARGO, O.A. Manganês em solos do Estado de São Paulo. R. Bras. Ci Solo, 7:123-130, 1983.

WEAR, J.L. \& SOMMER, A.L. Acid-extractable zinc of soils in relation to the occurrence of zinc deficiency symptoms of corn: a method analysis. Soil Sci. Soc. Am. J., 12:143-144. 1948 . 